Marquette University

e-Publications@Marquette

Mechanical Engineering Faculty Research and

Publications

Engineering, College of

4-1-2010

\title{
Reducing Orbit Covariance for Continuous Thrust Spacecraft Transfers
}

Scott Zimmer

Oscar Ocampo

Robert H. Bishop

Marquette University, robert.bishop@marquette.edu

Accepted version. IEEE Transactions on Aerospace and Electronic Systems, Vol. 46, No. 2 (April 2010):

771-791. DOI. (C) 2010 Institute of Electrical and Electronics Engineers (IEEE). Used with permission.

Robert H. Bishop was affiliated with the University of Texas at Austin at the time of publication. 


\title{
Reducing Orbit Covariance for Continuous Thrust Spacecraft Transfers
}

\author{
Scott Zimmer \\ Department of Aerospace Engineering and Engineering Mechanics, \\ University of Texas at Austin \\ Cesar Ocampo \\ Department of Aerospace Engineering and Engineering Mechanics, \\ University of Texas at Austin \\ Robert Bishop \\ Department of Aerospace Engineering and Engineering Mechanics, \\ University of Texas at Austin
}

\begin{abstract}
The calculus of variations is used to develop the necessary theory and derive the optimality conditions for a spacecraft to transfer between a set of initial and final conditions, while minimizing a combination of fuel consumption and a function of the estimation error covariance matrix associated with the spacecraft state. The theory is developed in a general manner that allows for multiple observers, moving observers, covariance associated with an arbitrary frame, a wide variety of observation types, multiple gravity bodies, and uncertainties in the spacecraft equations of motion based on the thrusting status of the engine. A series of example trajectories from low Earth orbit (LEO) to a near geosynchronous Earth orbit (GEO) shows that either the trace of the covariance at the final time or the integral of the trace of the covariance matrix associated with the error in the Cartesian position and velocity can be reduced significantly with a small increase in the fuel consumption. An additional example illustrates the covariance associated with the semimajor axis can be significantly reduced for a transfer from Earth orbit to lunar orbit. This example illustrates multiple, moving observers as well as a transfer in a multi-body gravitational field.
\end{abstract}

IEEE Transactions on Aerospace and Electronic Systems, Vol 46, No. 2 (April 2010): 771-791. DOI. This article is C Institute of Electrical and Electronics Engineers (IEEE) and permission has been granted for this version to appear in e-Publications@Marquette. Institute of Electrical and Electronics Engineers (IEEE) does not grant permission for this article to be further copied/distributed or hosted elsewhere without the express permission from Institute of Electrical and Electronics Engineers (IEEE). 


\section{SECTION I.}

\section{Nomenclature}

a Semimajor axis

A Derivative of $y$ state with respect to $\mathbf{x}$ state

B relates covariance before and after a discrete measurement

e Eccentricity

F Derivative of time derivative of Cartesian spacecraft position and velocity with respect to Cartesian spacecraft position and velocity

g Gravitational acceleration acting on spacecraft

G Derivative of time derivative of $y$ frame state with respect to $y$ frame state

$\mathrm{H}$ Derivative of observation with respect to spacecraft position and velocity

$H_{a}$ Hamiltonian function

$J$ One-half of the integral of spacecraft thrust acceleration squared

L Derivative of measurement constraint with respect to covariance

$m$ Spacecraft mass

$n$ Number of discrete times a function of the spacecraft covariance is included in the cost function

$\mathrm{n}$ Random vector of errors in observation

$p \quad$ Number of discrete measurements

$\mathrm{p}$ Vector from central body to observer

$P$ Power

P Covariance matrix for spacecraft position and velocity

$q$ Integral of a function of the spacecraft covariance

Q Spectral density matrix for errors in equations of motion

$r$ Radial position

$r$ Spacecraft position

$\mathbf{R}$ Spectral density matrix for observation noise

$\tilde{\mathbf{R}}$ Covariance matrix for observation noise

$s \quad$ Term that appears in the $L$ matrix

$t$ Time

$T_{1} \quad$ Error in thrust acceleration

$u$ Thrust acceleration magnitude

$\mathrm{u}$ Thrust direction unit vector

v Velocity

IV Random vector of errors in equations of motion

$W$ Cost function

$w_{1}^{2}$ Constant term in spectral density matrix for equations of motion

$x_{s} \quad x$ component of vector from observer to spacecraft 
x Cartesian frame

X Spacecraft Cartesian state including only position and velocity

$\tilde{\mathbf{X}}$ Spacecraft state including covariance

$y_{s} \mathrm{y}$ component of vector from observer to spacecraft

y Arbitrary frame

Y Spacecraft state in nonCartesian frame

$z$ Observation

$\alpha$ Thrust direction

$\alpha_{1}$ Error in thrust direction

$\alpha$ Free variables in planar orbit to orbit transfer

$\gamma \quad$ Lagrange multiplier

$\Gamma \quad$ Thrust acceleration magnitude

$\delta$ Dirac delta function

$\eta \quad$ Lagrange multiplier

$\theta$ Polar angle

$\theta$ Constraints on the spacecraft state at the initial time

$\kappa \quad$ True anomaly

$\lambda_{r} \quad$ Spacecraft radial position costate

$\lambda_{r} \quad$ Spacecraft position costate

$\lambda_{r} \quad$ Spacecraft radial velocity costate

$\lambda_{v} \quad$ Spacecraft velocity costate

$\lambda_{J} \quad$ Thrust acceleration costate

$\lambda_{\theta} \quad$ Spacecraft polar position costate

$\lambda_{\theta} \cdot$ Spacecraft polar velocity costate

$\lambda_{1-21}$ Spacecraft covariance costate

$\mu \quad$ Gravitational parameter of central body

$v$ Lagrange multiplier

$\xi$ Constant

$\rho$ Parameter vector used in constraints

$\sigma$ Constraint relating covariance before and after a discrete measurement

$\varphi$ Scalar weight

$\chi \quad$ Function of the covariance added to the cost function

$\psi$ Constraints on the spacecraft state at the final time

$\omega \quad$ Longitude of perigee. 


\author{
I. Subscripts \\ Initial \\ $f$ Final \\ Integrator \\ Maximum \\ Nominal \\ $r$ Radial \\ $s$ Prescribed \\ $t$ Cross-track \\ $x$ Associated with the $\mathbf{X}$ frame \\ Y Associated with the $\mathbf{Y}$ frame.
}

\title{
I. Superscripts
}

* True value

Targeted value

$\sim$ Augmented state including the covariance terms

$\wedge$ Function equivalent in different frame

Covariance terms or covariance costates associated with y frame.

\section{SECTION II.}

\section{Introduction}

The objective of this investigation is to demonstrate a technique that determines trajectories which minimize a combination of fuel consumption and spacecraft state uncertainty. Most current research first seeks a mass or time optimal transfer, followed by a second, independent study to determine if the optimal trajectory can be navigated. One must determine the number, quality, frequency, and types of measurements of the spacecraft state which must be taken in order to navigate the spacecraft with the required precision. Because no effort is made to improve observability during the optimization process, observability along the optimal trajectory may be significantly worse than the observability along another trajectory that has only a slightly greater fuel or time cost.

Betts provides an excellent summary of the techniques available for determining mass and time optimal trajectories. ${ }^{1}$ This investigation relied on indirect trajectory optimization techniques. ${ }^{2,3}$ Previous efforts to simultaneously improve the observability along a trajectory have been limited. In 1968 Vander Stoep presented a method of transferring from a specified initial state to a specified final state in a fixed period of time. ${ }^{4}$

IEEE Transactions on Aerospace and Electronic Systems, Vol 46, No. 2 (April 2010): 771-791. DOI. This article is (C) Institute of Electrical and Electronics Engineers (IEEE) and permission has been granted for this version to appear in e-Publications@Marquette. Institute of Electrical and Electronics Engineers (IEEE) does not grant permission for this article to be further copied/distributed or hosted elsewhere without the express permission from Institute of Electrical and Electronics Engineers (IEEE). 
During this transfer both the continuous observations and the equations of motion were corrupted by white noise. The cost function for this study was an integral of a function of the control, state, covariance, and time, combined with a weighted sum of elements of the Cartesian covariance matrix at the final time. In 1970 Schmidt extended Vander Stoep's method to allow free final time as well as both free and linearly constrained final states. ${ }^{5}$ This dissertation focused on determining the optimal controls for a closed-loop feedback control system instead of designing a trajectory to minimize open-loop estimation errors.

Between 1984 and 1990 Speyer et. al. and Hull et. al. presented a method of improving the observability in the homing missile problem by maximizing the trace of the Fisher information matrix. ${ }^{6-7,8}$ They showed that the likelihood of missile intercept in the homing missile problem could be increased by altering the intercept trajectory away from proportional navigation. In 1999 Oshman and Davidson presented a method of optimizing the trajectory of an observer who is attempting to determine the location of a fixed target by taking observations of the angle between the local horizontal and the target. ${ }^{9}$ The cost function in this study was the determinant of the Fisher information matrix plus a penalty function that was based on the observer location. In addition constraints were placed on the observer location. In this study the control was discretized, and an approximate optimal control was determined.

Between 1996 and 2004 Seywald investigated a technique to determine trajectories that were less sensitive to uncertainties in the initial spacecraft state. ${ }^{10-11,12}$ Seywald used the state transition matrix to estimate the first-order error in the final state caused by errors in the initial spacecraft state. He included these errors in the cost function, along with a measure of the fuel efficiency of the transfer. This technique would have provided the same results as the current study if the equations of motion were modeled without noise and if no measurements were employed. In 2005 Mishne applied the same technique as Seywald to optimally reconfigure a satellite formation when there is uncertainty in the initial state of the satellites. ${ }^{13}$

This study develops the optimality conditions for minimizing a combination of spacecraft state uncertainty and fuel consumption for an open-loop transfer between an arbitrary set of initial and final conditions. The third section develops the theory in a general manner that allows for multiple observation types, arbitrary gravity fields, discrete measurements, periods without measurements, and dynamic errors based on the thrust. The limited previous research into incorporating observability into trajectory optimization has all sought to minimize uncertainty associated with the spacecraft state in the integration frame. ${ }^{4-5,6,7,8,9,10,11,12,13}$ Because most trajectory optimization is done in a 
Cartesian frame, these techniques provide a method to minimize a combination of the propellant mass and the sum of the expected mean square errors in the spacecraft Cartesian position and velocity. Instead of, or in addition to, minimizing the covariance associated with the spacecraft Cartesian position and velocity, this work demonstrates a technique to minimize the covariance associated with nonCartesian frames, such as orbital elements or flight path angle.

The fourth section of this paper contains a series of example problems. The first four example problems are planar orbit to orbit transfer problems that illustrate the differences between continuous scalar measurements, alternating periods of continuous measurements and no measurements, discrete measurements, and dynamic errors based on the spacecraft thrusting status. The final example involves a transfer from near geosynchronous Earth orbit (GEO) to a low lunar orbit, where the covariance associated with the semimajor axis in the target orbit is minimized. Minimizing the covariance associated with the semimajor axis is especially interesting because the error in estimating the spacecraft state at some time after the spacecraft is inserted into the target orbit is dominated by the error in the spacecraft semimajor axis when it is inserted into the target orbit. ${ }^{14}$ This example demonstrates that the covariance associated with the semimajor axis can be reduced significantly with only a slight increase in fuel consumption.

\section{SECTION III.}

\section{General Theory}

\section{A. Problem formulation}

In order to use the calculus of variations to optimize a trajectory, a scalar cost function must be defined to measure the trajectory's merit. ${ }^{15}$ Because the goal is to maximize a combination of the observability along a trajectory and the trajectory's fuel efficiency, the cost function must include both mass and observability terms. Improving the observability along a trajectory is important because the ability to deliver the spacecraft to the target orbit or constraint is dependent on how well the spacecraft state can be estimated. for a mass-optimal solution, the choice of cost function is straightforward, with the mass of the spacecraft at the final time providing one obvious choice. Determining a scalar representation of the observability along a trajectory is more difficult. Bishop et. al. presented a method based on the inverse function theorem to estimate the state observability along a trajectory using the determinant of the observability matrix. ${ }^{16}$ This measure was rejected for this study because its effectiveness in measuring the 
observability along a trajectory is relatively unknown, and it yields an abstruse function that must be incorporated in the cost function.

The observability term for this study is a function of the estimation error covariance matrix associated with the spacecraft position and velocity because it provides an estimate of the mean square errors in the spacecraft position and velocity as well as the correlation between the errors in different components of the position and velocity vectors. A general cost-function-incorporating covariance is given by (1), where $\varepsilon_{i}$ is an arbitrary function of the covariance, position, and velocity at discrete times, $t_{i}$ and where $\chi$ is an arbitrary function of covariance, position, and velocity. The choice of $\varepsilon_{i}$ and $t_{i}$ allow one to minimize a given function of the covariance at any discrete time(s), such as immediately before an impulsive maneuver, a gravity assist, or an atmospheric entry. The function $\chi$ allows one to minimize the integral of a function of the covariance along the trajectory. Note that the covariance matrix included in the cost function is the covariance associated with Cartesian position and velocity. It is possible to select the functions $\varepsilon$ and $\chi$ so the covariance associated with another coordinate frame, such as orbital elements, is included in the cost function.

$$
\begin{gathered}
W=J\left(t_{f}\right)+\sum_{i=1}^{n} \varepsilon_{i}\left[\mathbf{r}\left(t_{i}\right), \mathbf{v}\left(t_{j}\right), \mathbf{P}\left(t_{i}\right)\right] \\
+\int_{t_{0}}^{t_{f}} \chi[\mathbf{r}(t), \mathbf{v}(t), \mathbf{P}(t)] d t
\end{gathered}
$$

The mass term in the cost function $J$ is defined to be one-half the integral of the thrust acceleration squared as specified by (2). for a power limited propulsion (PLP) engine, minimizing $J$ is equivalent to maximizing the final mass since $J$ and the fuel required are ordered and one-to-one. ${ }^{17}$ The mass at any time can be determined from the initial mass and the power available to the engine by using (3). Utilizing $J$ instead of mass, allows one to solve optimization problems when the initial spacecraft mass or the power available to the engine is not known. One can make this PLP formulation mimic a constant specific impulse formulation by constraining either the engine's thrust or specific impulse. The variable $q$ is defined to be the integral of $\chi$ as shown in (4). 


$$
\begin{aligned}
J(t) & =\int_{t_{0}}^{t} \frac{\Gamma^{2}}{2} d t \\
m(t) & =\frac{m\left(t_{0}\right) P_{\max }}{P_{\max }+m\left(t_{0}\right) J(t)} \\
q(t) & =\int_{t_{0}}^{t} \chi[\mathbf{r}(t), \mathbf{v}(t), \mathbf{P}(t)] d t .
\end{aligned}
$$

$(2)(3)(4)$

In order to make the cost function depend only on the state, time, and constant parameters, define the augmented state $\tilde{X}$ as in (5), where $P_{i}$ are defined in (6). The covariance terms are only associated with the position and velocity states and are defined in (6) for the three dimensional case. Because the covariance matrix is symmetric, there are only 21 unique elements for the three dimensional case.

$$
\begin{aligned}
\tilde{\mathbf{X}}^{T} & =\left(\mathbf{r}^{T} \mathbf{v}^{T} J P_{1} \ldots P_{21} q\right) . \\
\mathbf{P}_{X} \equiv & \left(\begin{array}{ccccccc}
P_{x x} & P_{x y} & P_{x z} & P_{x x} & P_{x y} & P_{x z} \\
P_{x y} & P_{y y} & P_{y z} & P_{y x} & P_{y y} & P_{y z} \\
P_{x z} & P_{y z} & P_{z z} & p_{z x} & p_{z y} & p_{z z} \\
P_{x x} & P_{y x} & P_{z x} & P_{x x} & P_{x y} & P_{x z} \\
P_{x y} & P_{y y} & P_{z y} & P_{x y} & P_{y y} & P_{y z} \\
P_{x z} & P_{y z} & P_{z z} & P_{x z} & P_{y z} & P_{z z} \\
x z & p_{2} & p_{3} & p_{4} & p_{5} & p_{6} \\
p_{1} & p_{2} & p_{8} & p_{9} & p_{10} & p_{11} \\
p_{2} & p_{7} & p_{8} & p_{13} & p_{14} & p_{15} \\
p_{3} & p_{8} & p_{12} & p_{13} \\
p_{4} & p_{9} & p_{13} & p_{16} & p_{17} & p_{18} \\
p_{5} & p_{10} & p_{14} & p_{17} & p_{19} & p_{20} \\
p_{6} & p_{11} & p_{15} & p_{18} & p_{20} & p_{21}
\end{array}\right) .
\end{aligned}
$$

$(5)(6)$ 
The time derivatives of the spacecraft position and velocity are given by (7) and (8). In order to find the time derivative of the covariance matrix, the linearized time derivative of the position and velocity, given by (9), is needed. The noise in the equations of motion is accounted for by $\mathbf{w}$, which is a zero-mean Gaussian white noise process with spectral density $\mathbf{Q}$. The model for the observation is given by (10). The noise in the observation measurement is accounted for by $\mathbf{n}$, which is a zero-mean Gaussian white noise process with spectral density $\mathbf{R}$. The time derivative of the covariance for periods of continuous measurements is given by the matrix Riccati equation given in (11). ${ }^{18}$ If no measurements are being taken, the time derivative of the covariance is given by (12). The relationship between the covariance after a discrete measurement and the covariance before the measurement is given by (13), where $t_{k}$ is the time of the measurement. ${ }^{18}$ The covariance at any time can be determined from the initial value of the covariance and from (11) to (13).

$$
\begin{aligned}
\mathbf{r}= & \mathbf{v} \\
\mathbf{v} & =\mathbf{g}(\mathbf{r})+\Gamma \mathbf{u} \\
\left(\begin{array}{c}
\cdot \\
\mathbf{r} \\
\mathbf{v}
\end{array}\right)= & \mathbf{F}\left(\begin{array}{l}
\mathbf{r} \\
\mathbf{v}
\end{array}\right)+\mathbf{w}, \mathbf{F}=\left.\frac{\partial\left(\begin{array}{l}
\mathbf{r} \\
\mathbf{v}
\end{array}\right)}{\partial(\mathbf{r v})}\right|_{\mathbf{r}, \mathbf{v}=\mathbf{r}_{\text {nom }}, \mathbf{v}_{\text {nom }}} \\
\mathbf{z}= & \mathbf{H}\left(\begin{array}{l}
\mathbf{r} \\
\mathbf{v}
\end{array}\right)+\mathbf{n}, \mathbf{H}=\left.\frac{\partial \mathbf{z}(\mathbf{r}, \mathbf{v})}{\partial(\mathbf{r v})}\right|_{\mathbf{r}, \mathbf{v}=\mathbf{r}_{\text {nom }}, \mathbf{v}_{\text {nom }}} \\
\cdot & \mathbf{P P}+\mathbf{P F}+\mathbf{Q}-\mathbf{P H} \mathbf{R}^{T} \mathbf{R}_{\mathbf{H}} \\
\cdot \cdot \mathbf{P}= & \mathbf{F P}+\mathbf{P F}+\mathbf{Q} \\
\boldsymbol{\sigma} \equiv & \mathbf{P}\left(t_{k+}\right)-\mathbf{P}\left(t_{k-}\right)+\mathbf{P}\left(t_{k-}\right) \mathbf{H}^{T}\left(t_{k}\right) \\
& {\left[\mathbf{H}\left(t_{k}\right) \mathbf{P}\left(t_{k-}\right) \mathbf{H}^{T}\left(t_{k}\right)+\tilde{\mathbf{R}}^{-1} \mathbf{H}\left(t_{k}\right) \mathbf{P}\left(t_{k-}\right)\right.} \\
& \mathbf{0 .}
\end{aligned}
$$

$(7)(8)(9)(10)(11)(12)(13)$

It is important to note that both $\mathrm{Q}$ and $\mathrm{R}$ may be functions of the time, spacecraft state, or controls. Although extended Kalman filter theory does not allow these spectral densities to be a function of the spacecraft state or control, empirical evidence suggests that this model is valid. The values of spectral density matrix $\mathrm{R}$ are based on the type of 
measurements taken, sensors used, and other factors related to a specific mission. for a discrete measurement $\tilde{\mathrm{R}}$ is the covariance associated with the errors in the observation measurements. The values of the spectral density matrix $Q$ are determined by how well the dynamic equations of motion are modeled as well as how accurately the engine can produce the desired thrust level.

The spectral density matrices $Q$ and $R$ and the initial covariance $\mathrm{P}\left(t_{0}\right)$ must be determined a priori. Only the relative value of these matrices is important because they can be scaled by the same factor without altering the solutions obtained from the optimization process. Because the covariance throughout the trajectory is scaled by that factor, the functions $\epsilon$ and $\chi$ should be divided by that same factor.

The time derivative of the full spacecraft state can easily be determined from (2), (4) (7), (8), (11), (12), and (13). The costates associated with the state defined by (5) are specified in (14). The Hamiltonian $H_{a}$ can be determined using the equations for the time derivative of the state and (15).

$$
\begin{aligned}
\lambda^{T} & =\left(\lambda_{\mathbf{r}}^{T} \lambda_{\mathbf{v}}^{T} \lambda_{J} \lambda_{1} \ldots \lambda_{21} \lambda_{q}\right)^{T} \\
H_{a} & =\lambda^{T} \tilde{\mathbf{x}}
\end{aligned}
$$

The optimal control problem is to transfer the spacecraft from an initial state constraint, given by (16), to the final state constraint, given by (17). The constraints may or may not include parameters $\rho$. Note the constraint may specify a prescribed initial and final orbit, a prescribed initial and final state, or some other function of the initial and final states. These constraints are adjoined to the cost function to form the augmented performance index in (18).

$(16)(17)(18)$

$$
\begin{aligned}
\theta\left(t_{0}, x_{0}, \rho\right) & =\mathbf{0} \\
\psi\left(t_{f}, x_{f}, \rho\right) & =\mathbf{0} \\
W^{\prime} & =W+v^{T} \theta+\eta^{T} \psi .
\end{aligned}
$$

The first variation of the augmented performance index is given by (19), where the $\sim$ above the $\delta$ symbolizes the time fixed variation. Requiring the first variation of $W^{\prime}$ to be 
zero in order to minimize $W^{\prime}$ yields the conditions in (20) to (28). These equations, along with (16) and (17), form the constraints required to set up a two point boundary value problem (TPBVP). If the covariance associated with the error in the linearized dynamics and measurements is independent of the controls, then (21) and (22) take on the familiar form of (29) and (30).

IEEE Transactions on Aerospace and Electronic Systems, Vol 46, No. 2 (April 2010): 771-791. DOI. This article is @ Institute of Electrical and Electronics Engineers (IEEE) and permission has been granted for this version to appear in e-Publications@Marquette. Institute of Electrical and Electronics Engineers (IEEE) does not grant permission for this article to be further copied/distributed or hosted elsewhere without the express permission from Institute of Electrical and Electronics Engineers (IEEE). 
NOT THE PUBLISHED VERSION; this is the author's final, peer-reviewed manuscript. The published version may be accessed by following the link in the citation at the bottom of the page.

$$
\begin{aligned}
& \delta W^{\prime}=\quad\left(\frac{\partial W^{\prime}}{\partial \tilde{\mathbf{X}}_{f}}-\lambda_{f}^{T}\right) \delta \tilde{\mathbf{X}}_{f}+\left[H_{a}\left(t_{f}\right)+\frac{\partial W^{\prime}}{\partial t_{f}}\right] \delta t_{f} \\
& +\left(\frac{\partial W^{\prime}}{\partial \tilde{\mathbf{X}}_{0}}+\lambda_{0}^{T}\right) \delta \tilde{\mathbf{X}}_{0}+\left[H_{a}\left(t_{0}\right)-\frac{\partial W^{\prime}}{\partial t_{0}}\right] \delta t_{0} \\
& +\frac{\partial W^{\prime}}{\partial \rho} \delta \rho+\sum_{i=1}^{n}\left[\frac{\partial \varepsilon_{i}}{\partial \tilde{\mathbf{X}}\left(t_{i}\right)}-\lambda\left(t_{i-}\right)+\lambda\left(t_{i+}\right)\right] \\
& \times \delta \tilde{\mathbf{X}}\left(t_{i}\right)_{t_{i} \neq t_{f}}+\int_{t_{0}}^{t_{f}}\left\{\left(\frac{\partial H_{a}}{\partial \tilde{\mathbf{X}}}+\lambda^{T}\right) \tilde{\delta} \tilde{\mathbf{X}}\right. \\
& \left.+\frac{\partial H_{a}}{\partial \Gamma} \tilde{\delta} \Gamma+\frac{\partial H_{a}}{\partial \mathbf{u}} \tilde{\delta} \mathbf{u}\right\} d t \\
& H_{a}\left(t_{0}\right)=\frac{\partial W^{\prime}}{\partial t_{0}} \\
& \frac{\partial H_{a}}{\partial \Gamma}=0 \\
& \frac{\partial H_{a}}{\partial \mathbf{u}}=0 \\
& \frac{\partial W^{\prime}}{\partial \tilde{\mathbf{x}}_{f}}=\lambda_{f}^{T} \\
& \partial \mathbf{X}_{f} \\
& H_{a}\left(t_{f}\right) \quad=-\frac{\partial W^{\prime}}{\partial t_{f}} \\
& \lambda_{0}^{T} \quad=-\frac{\partial W^{\prime}}{\partial \tilde{\mathbf{X}}_{0}} \\
& \frac{\partial W^{\prime}}{\partial \rho} \quad=0 \\
& \frac{\partial \varepsilon_{i}}{\partial \tilde{\mathbf{X}}\left(t_{i}\right)}-\lambda\left(t_{i-}\right)+\lambda\left(t_{i+}\right)=\left.0\right|_{i=1 \rightarrow n} \\
& \lambda^{T} \quad=-\frac{\partial H_{a}}{\partial \tilde{\mathbf{X}}} \\
& \mathbf{u} \quad \frac{=\lambda_{\mathbf{v}}}{\lambda_{\mathbf{v}}} \\
& \Gamma \quad=-\frac{\lambda_{v}}{\lambda_{J}} \text {. }
\end{aligned}
$$

IEEE Transactions on Aerospace and Electronic Systems, Vol 46, No. 2 (April 2010): 771-791. DOI. This article is (C) Institute of Electrical and Electronics Engineers (IEEE) and permission has been granted for this version to appear in e-Publications@Marquette. Institute of Electrical and Electronics Engineers (IEEE) does not grant permission for this article to be further copied/distributed or hosted elsewhere without the express permission from Institute of Electrical and Electronics Engineers (IEEE). 


\section{B. Discrete Measurements}

Because the optimality conditions derived with the calculus of variations require the covariance matrix to be included in the spacecraft state, each discrete observation provides a discontinuity in the spacecraft state. Consequently the constraint that relates the covariance after the measurement to the covariance before the measurement must be included in the cost function, as shown in (31). The summation indicates that the covariance must be constrained at each of the $p$ measurements. This discontinuity in the spacecraft state causes the optimal costates to be discontinuous across each measurement as well. Because discrete observations each add either 10 constraints for a planar transfer or 21 constraints for a three dimensional transfer, the state will have 10 or 21 times the number of observations discontinuities. These constraints require that the unique elements of the covariance matrix satisfy (13). While these jumps do not provide theoretical difficulty, they do make it more difficult to numerically find solutions to the problem.

$$
\begin{aligned}
W^{\prime}= & J\left(t_{f}\right)+\sum_{i=1}^{n} \varepsilon_{i}\left[\mathbf{x}\left(t_{i}\right), \mathbf{P}_{x}\left(t_{i}\right)\right] \\
& +\int_{t_{0}}^{t_{f}} \chi\left[\mathbf{x}(t), \mathbf{P}_{x}(t)\right] d t+v^{T} \boldsymbol{\theta}+\boldsymbol{\eta}^{T} \psi+\sum_{k=1}^{p}\left(\gamma^{T} \boldsymbol{\sigma}\right)_{k} .
\end{aligned}
$$

The costates before and after each measurement must satisfy (32) to (36). Because of the formulation of the constraints in (13), where $\mathrm{P}\left(t_{k+}\right)$ appears linearly, the covariance costates after the measurement must satisfy (37). Consequently one can formulate the constraint expressed in (38), where the L matrix is defined in (39). The covariance costates after each measurement can be uniquely determined from the covariance costates before the measurement and from the augmented spacecraft state $\tilde{X}$ as long as the L matrix can be inverted. If the $\mathrm{L}$ matrix is singular, then there exist an infinite number of possible values for the covariance costates after the measurement that satisfy the first-order extremal conditions. As a result no unique solution exists for the TPBVP if L is singular. 
NOT THE PUBLISHED VERSION; this is the author's final, peer-reviewed manuscript. The published version may be accessed by following the link in the citation at the bottom of the page.

$$
\begin{aligned}
\lambda_{i}\left(t_{k-}\right) & =\frac{\partial W}{\partial P_{i}\left(t_{k-}\right)} \\
\lambda_{i}\left(t_{k+}\right) & =-\frac{\partial W}{\partial P_{i}\left(t_{k+}\right)} \\
\lambda_{r}\left(t_{k+}\right)-\lambda_{r}\left(t_{k-}\right)+\frac{\partial W}{\partial \mathbf{r}\left(t_{k}\right)} & =\mathbf{0} \\
\lambda_{v}\left(t_{k+}\right)-\lambda_{v}\left(t_{k-}\right)+\frac{\partial W}{\partial \mathbf{v}\left(t_{k}\right)} & =\mathbf{0} \\
\lambda_{J}\left(t_{k+}\right) & =\lambda_{J}\left(t_{k-}\right) \\
\lambda_{i}\left(t_{k+}\right) & =-\gamma_{i} \\
\mathbf{L} \lambda_{1-21}\left(t_{k+}\right) & =\lambda_{1-21}\left(t_{k-}\right) \\
\mathbf{L}(i, j) & =\frac{\partial \sigma_{j}}{\partial P_{i}}
\end{aligned}
$$

$(32)(33)(34)(35)(36)(37)(38)(39)$

In order to verify the existence of $\mathrm{L}^{-1}$, one could, alternatively express the constraints from (13) as the constraints in (40), where the B matrix is defined in (41). If the B matrix is invertible, then the $\mathrm{L}$ matrix must also be invertible. Consider the case where the solution to the TPBVP is sought by integrating backwards in time. At the time just after the last measurement, the entire augmented state and costate are known. The position, velocity, and integral of thrust acceleration squared do not change during the measurement, and the covariance before the measurement can be determined by inverting B in (40). The covariance costates before the measurement can be determined from (38) and the other costates before the measurement can be determined from (34) to (36). This known, complete set of states and costates can then be integrated back in time until the time of the previous measurement, and the process can be repeated until the initial time is reached. The set of optimal costates are unique in this solution. Because the solution is unique when the TPBVP is solved by integrating backwards in time, the costates must be unique when integrating forward in time, and the inverse of L must exist.

$(40)(41)$

$$
\begin{aligned}
\mathbf{P}\left(t_{k+}\right) & =\mathbf{B}\left(t_{k+}\right) \mathbf{P}\left(t_{k-}\right) \\
\mathbf{B} & \equiv \mathbf{I}-\mathbf{P}\left(t_{k+}\right) \mathbf{H}^{T}\left(t_{k}\right) \tilde{\mathbf{R}}^{-1} \mathbf{H}\left(t_{k}\right) .
\end{aligned}
$$

IEEE Transactions on Aerospace and Electronic Systems, Vol 46, No. 2 (April 2010): 771-791. DOI. This article is @ Institute of Electrical and Electronics Engineers (IEEE) and permission has been granted for this version to appear in e-Publications@Marquette. Institute of Electrical and Electronics Engineers (IEEE) does not grant permission for this article to be further copied/distributed or hosted elsewhere without the express permission from Institute of Electrical and Electronics Engineers (IEEE). 


\section{Dynamic Errors Based on Thrust}

Errors in the thrust magnitude and direction contribute significantly to the errors in the dynamic equations of motion. These engine thrust errors are more significant when the engine is providing more thrust. This application allows the spectral density of the errors in the dynamics w from (9) to be functions of the thrust magnitude and direction. The resulting optimal control depends on whether the controls are modeled as separate engines, each of which provides thrust acceleration in one of the coordinate directions, or as a single engine and two angles, which provide the overall three-dimensional thrust.

In the former case the errors in the thrust magnitudes for each engine are assumed to be independent. As a result $w$ can be written as in (42), where $\omega_{x}, \omega_{y}$, and $\omega_{z}$ account for the errors in the dynamic equations due to gravitational terms, solar winds, and other forces which are not modeled. They have zero-mean, are independent, and have spectral density $\omega_{1}^{2}$. The errors in the thrust acceleration $T_{1}, T_{2}$, and $T_{3}$ are also zero-mean and independent of each other and $\omega$. These errors are modeled as dependent on the magnitude of the thrust acceleration provided by each engine, with spectral density specified by (43) to (45), where $E$ is the expectation operator. The spectral density Q can now be written as (46). 


$$
\begin{aligned}
& \mathbf{w}(t)=\left(\begin{array}{l}
\omega_{x} \\
\omega_{y} \\
\omega_{z}
\end{array}\right)+\left(\begin{array}{l}
T_{1} \\
T_{2} \\
T_{3}
\end{array}\right) \\
& \frac{E\left[T_{1}(t) T_{1}(\tau)\right]}{\delta(t-\tau)}=\frac{\Gamma_{x}^{2}}{\xi_{1}} \\
& \frac{E\left[T_{2}(t) T_{2}(\tau)\right]}{\delta(t-\tau)}=\frac{\Gamma_{y}^{2}}{\xi_{2}} \\
& \frac{E\left[T_{3}(t) T_{3}(\tau)\right]}{\delta(t-\tau)}=\frac{\Gamma_{z}^{2}}{\xi_{3}} \\
& \mathbf{Q}(t)=\frac{E\left[\mathbf{w}(t) \mathbf{w}^{T}(\tau)\right]}{\delta(t-\tau)} \\
& =\left(\begin{array}{cccccc}
0 & 0 & 0 & 0 & 0 & 0 \\
0 & 0 & 0 & 0 & 0 & 0 \\
0 & 0 & 0 & 0 & 0 & 0 \\
0 & 0 & 0 & w_{1}^{2}+\frac{\Gamma_{x}^{2}}{\xi_{1}} & 0 & 0 \\
0 & 0 & 0 & 0 & w_{1}^{2}+\frac{\Gamma_{y}^{2}}{\xi_{2}} & 0 \\
0 & 0 & 0 & 0 & 0 & w_{1}^{2}+\frac{\Gamma_{z}^{2}}{\xi_{3}}
\end{array}\right) .
\end{aligned}
$$

$(42)(43)(44)(45)(46)$

The first-order optimality conditions require that the derivative of the Hamiltonian with respect to $\Gamma_{X}, \Gamma_{y}$, and $\Gamma_{z}$ be zero. The terms in the Hamiltonian involving $\Gamma_{x}, \Gamma_{y}$, and $\Gamma_{z}$ are given by (47). The thrust acceleration along the optimal trajectory is then given by (48). Notice the similarity between (29), (30), and (48). If the error in the dynamic equations is not related to the magnitude of the thrust acceleration, then $\xi_{1}, \xi_{2}$, and $\xi_{3}$ would be infinite and the thrust acceleration terms in (48) would be unchanged from the values in (29) and (30).

IEEE Transactions on Aerospace and Electronic Systems, Vol 46, No. 2 (April 2010): 771-791. DOI. This article is (C) Institute of Electrical and Electronics Engineers (IEEE) and permission has been granted for this version to appear in e-Publications@Marquette. Institute of Electrical and Electronics Engineers (IEEE) does not grant permission for this article to be further copied/distributed or hosted elsewhere without the express permission from Institute of Electrical and Electronics Engineers (IEEE). 
$(47)(48)$

$$
\begin{aligned}
H_{a}= & \lambda_{v x} \Gamma_{x}+\lambda_{v y} \Gamma_{y}+\lambda_{v z} \Gamma_{z}+\lambda_{J} \frac{\Gamma_{x}^{2}+\Gamma_{y}^{2}+\Gamma_{z}^{2}}{2} \\
& +\lambda_{16} \frac{\Gamma_{x}^{2}}{\xi_{1}}+\lambda_{19} \frac{\Gamma_{y}^{2}}{\xi_{2}}+\lambda_{21} \frac{\Gamma_{z}^{2}}{\xi_{3}}+\text { non }{ }^{2} \text { terms } \\
\Gamma_{x}= & \frac{-\lambda_{v x}}{\lambda_{J}+\frac{2 \lambda_{16}}{\xi_{1}}} \Gamma_{y}=\frac{-\lambda v y}{\lambda_{J}+\frac{2 \lambda_{19}}{\xi_{2}}} \Gamma_{z}=\frac{-\lambda_{v z}}{\lambda_{J}+\frac{2 \lambda_{21}}{\xi_{3}}} .
\end{aligned}
$$

In most applications separate engines are not used to create thrust in the three coordinate axes. In these cases the errors in the dynamic equations of motion will depend on the difference between the actual thrust acceleration magnitude and the desired thrust acceleration magnitude as well as errors in the direction of the thrust acceleration. Consider a two dimensional example, where the controls are the magnitude of the thrust acceleration $\Gamma$ and the thrust direction measured from the x-axis $\alpha$. The noise in the equations of motion is specified by (49), where $*$ indicates an actual value provided by the engine and where $\&$ indicates a prescribed value. The difference between the actual thrust acceleration magnitude and direction and the prescribed values is given by (50), where both $T_{1}$ and $\alpha_{1}$ are zero-mean and independent, with spectral density given by (51). Because both $T_{1}$ and $\alpha_{1}$ are small, terms involving $T_{1}$ multiplied by $\alpha_{1}$ or $\alpha_{1}^{2}$ are ignored. As a result (49) can be simplified as (52) by using a Taylor Series expansion. The spectral density Q can now be written as in (53). Note that the expected error in the thrust acceleration in the coordinate directions is coupled in this formulation unlike the previous formulation, that assumed separate engines in the component directions. 


$$
\begin{aligned}
& \mathbf{w}(t) \quad=\left(\begin{array}{l}
\omega_{x} \\
\omega_{y}
\end{array}\right)+\Gamma^{*}\left[\begin{array}{c}
\cos \left(\alpha^{*}\right) \\
\sin \left(\alpha^{*}\right)
\end{array}\right]-\Gamma^{\&}\left[\begin{array}{c}
\cos \left(\alpha^{\&}\right) \\
\sin \left(\alpha^{\&}\right)
\end{array}\right] \\
& \Gamma^{*} \quad=\Gamma^{\&}+T_{1}, \alpha^{*}=\alpha^{\&}+\alpha_{1} \\
& \frac{E\left[T_{1}(t) T_{1}(\tau)\right]}{\delta(t-\tau)}=\frac{\left(\Gamma^{\&}\right)^{2}}{\xi_{1}} \frac{E\left[\alpha_{1}(t) \alpha_{1}(\tau)\right]}{\delta(t-\tau)}=\frac{1}{\xi_{2}} \\
& \mathbf{w}(t) \\
& =\left(\begin{array}{l}
\omega_{X} \\
\omega_{y}
\end{array}\right)+\frac{T_{1}}{\Gamma^{*}}\left(\begin{array}{l}
\Gamma_{x} \\
\Gamma_{y}
\end{array}\right)+\alpha_{1}\left(\begin{array}{c}
-\Gamma_{y} \\
\Gamma_{x}
\end{array}\right) \\
& \mathbf{Q}(t) \\
& =\frac{E\left[\mathbf{w}(t) \mathbf{w}^{T}(\tau)\right]}{\delta(t-\tau)} \\
& =\left(\begin{array}{cccc}
0 & 0 & 0 & 0 \\
0 & 0 & 0 & 0 \\
0 & 0 & w_{1}^{2}+\frac{\Gamma_{x}^{2}}{\xi_{1}}+\frac{\Gamma_{y}^{2}}{\xi_{2}} & \Gamma_{x} \Gamma_{y}\left(\frac{1}{\xi_{1}}-\frac{1}{\xi_{2}}\right) \\
0 & 0 & \Gamma_{x} \Gamma_{y}\left(\frac{1}{\xi}-\frac{1}{\xi_{2}}\right) & w_{1}^{2}+\frac{\Gamma_{x}^{2}}{\xi_{2}}+\frac{\Gamma_{y}^{2}}{\xi}
\end{array}\right) .
\end{aligned}
$$

The terms in the Hamiltonian involving $\Gamma_{x}$ and $\Gamma_{y}$ are given by (54). The first-order optimality conditions again require that the derivative of the Hamiltonian, with respect to $\Gamma_{X}$ and $\Gamma_{y}$, be zero. The optimal thrust acceleration is then given by (55). Again (55) reduces to (29) and (30), when the error in the thrust acceleration is independent of the thrust magnitude and direction. 
NOT THE PUBLISHED VERSION; this is the author's final, peer-reviewed manuscript. The published version may be accessed by following the link in the citation at the bottom of the page.

$$
\begin{aligned}
H_{a} & =\lambda_{v x} \Gamma_{x}+\lambda_{v y} \Gamma_{y}+\lambda_{J} \frac{\Gamma_{x}^{2}+\Gamma_{y}^{2}}{2}+\lambda_{8}\left(\frac{\Gamma_{x}^{2}}{\xi_{1}}+\frac{\Gamma_{y}^{2}}{\xi_{2}}\right) \\
& +\lambda_{9} \Gamma_{x} \Gamma_{y}\left(\frac{1}{\xi_{1}}-\frac{1}{\xi_{2}}\right)+\lambda_{10}\left(\frac{\Gamma_{x}^{2}}{\xi_{2}}+\frac{\Gamma_{y}^{2}}{\xi_{1}}\right) \\
\Gamma_{y} & =-\frac{\lambda_{v y}-\lambda_{9} \lambda_{v x} d\left(\frac{1}{\xi_{1}}-\frac{1}{\xi_{2}}\right)}{\lambda_{J}+2\left(\frac{\lambda_{10}}{\xi_{1}}+\frac{\lambda_{8}}{\xi_{2}}\right)-\lambda_{9}^{2} d\left(\frac{1}{\xi_{1}}-\frac{1}{\xi_{2}}\right)^{2}} \\
\Gamma_{x} & =-d\left[\lambda_{v x}+\lambda_{9} \Gamma_{y}\left(\frac{1}{\xi_{1}}-\frac{1}{\xi_{2}}\right)\right] \\
d & =\left[\lambda_{J}+2\left(\frac{\lambda_{8}}{\xi_{1}}+\frac{\lambda_{10}}{\xi_{2}}\right)\right]^{-1} .
\end{aligned}
$$

$(54)(55)$

\section{Minimizing Covariance in Alternate Frames}

Instead of minimizing the covariance associated with the spacecraft Cartesian position and velocity, defined to be the $\mathbf{x}$ frame, one may wish to minimize a function of the covariance associated with the spacecraft orbital elements, or any other nonCartesian frame, defined to be the $y$ frame, that can be expressed as in (56). The cost function, given by (57), allows one to minimize a function of the covariance along the trajectory as well as at discrete times. One technique to employ the calculus of variations to determine an optimal trajectory with this cost function, which includes the covariance associated with the $y$ frame, requires the entire problem be formulated in the $y$ frame. This formulation requires that the spacecraft equations of motion, time derivative of the covariance matrix, time derivatives of the costates, and optimal values of the costates be derived in the $y$ frame. Alternatively one could express the y frame covariance using (58), where $\mathbf{A}$ is the derivative of the $y$ state with respect to the $x$ state. This derivative must be continuous with a continuous inverse and must not be an explicit function of time in order to use the following technique. Because $\mathbf{A}$ and $\mathbf{y}$ can be written as functions of $\mathbf{x}$, the cost function from (57) can be expressed as (59). with (59) as the cost function, the entire problem can be solved in the $\mathbf{x}$ frame without deriving equations of motion or optimality conditions in the $y$ frame. Although linear transformations are often used to approximate the covariance in another frame at a particular time ${ }^{19}$ proves that the orbit obtained using the linear

IEEE Transactions on Aerospace and Electronic Systems, Vol 46, No. 2 (April 2010): 771-791. DOI. This article is @ Institute of Electrical and Electronics Engineers (IEEE) and permission has been granted for this version to appear in e-Publications@Marquette. Institute of Electrical and Electronics Engineers (IEEE) does not grant permission for this article to be further copied/distributed or hosted elsewhere without the express permission from Institute of Electrical and Electronics Engineers (IEEE). 
transformation and cost function in (58) and (59) is the exact same orbit obtained by formulating the problem in the alternate frame, as long as the linear matrix Riccati equation is utilized for covariance propagation.

$$
\begin{aligned}
\mathbf{y} & =\mathbf{s}(\mathbf{x}) \\
W & =J_{f}+\sum_{i=1}^{n} \varepsilon_{i}\left[\mathbf{y}\left(t_{i}\right), \mathbf{P}_{y}\left(t_{i}\right)\right]+\int_{t_{0}}^{t_{f}} \chi\left[\mathbf{y}(t), \mathbf{P}_{y}(t)\right] d t \\
\mathbf{P}_{y} & =\mathbf{A P}_{x} \mathbf{A}^{T} \\
W & =J_{f}+\sum_{i=1}^{n} \varepsilon_{i}\left[\mathbf{x}\left(t_{i}\right), \mathbf{P}_{x}\left(t_{i}\right)\right]+\int_{t_{0}}^{t_{f}} \hat{\imath}\left[\mathbf{x}(t), \mathbf{P}_{x}(t)\right] d t
\end{aligned}
$$

$(56)(57)(58)(59)$

\section{E. Solution Process}

The equations in the previous sections provide a general method to determine optimal trajectories between any initial and final constraints. The solution process sets up a TPBVP, where the unknowns are the parameters associated with the initial state and the initial costates. The constraints restrict the final state and costate. Note the term optimal means trajectories that maximize a combination of fuel efficiency and trajectory observability. By varying the relative values of the terms in the cost function, either fuel efficiency or observability can be emphasized.

A major difficulty associated with solving this type of TPBVP is the sensitivity of the solution process to the initial estimate of the costates. One might assume that including observability in the cost function makes determining an initial estimate quite difficult since including observability requires the estimate of 14 initial costates instead of 4 initial costates for the mass optimal solution in two dimensions or 27 costates instead of 6 in three dimensions. In fact it is no more difficult to estimate the costates with observability than without observability. The first step of the solution process can always be to solve the mass optimal problem and to obtain the value of the costates for that problem. This solution, with all of the covariance costates set to zero, is the solution if the observability terms in the cost function are multiplied by a scalar weight of zero. This solution, with the scalar weight of zero, is an excellent initial guess for a problem where the observability terms are multiplied by a small scalar weight. The solution with a small scalar weight can 
then be utilized as the initial guess for a larger scalar weight. By slowly increasing these scalar weights for the observability terms in the cost function, one can 'walk' a mass optimal solution to a solution that optimizes for observability and fuel efficiency without needing to guess the initial costates for the covariance terms. Using the state transition matrix to compute derivatives instead of finite differences provides a faster, more robust method to 'walk' from solutions that ignore observability to ones that incorporate observability. ${ }^{20,21}$

\section{SECTION IV.}

\section{Example Problems}

\section{A. Planar Orbit to Orbit Transfer Optimality Conditions}

The first four example applications are planar transfers through a central body gravity field specified by (60) from an initial orbit with eccentricity $e_{0}$ semimajor axis $a_{0}$, and argument of perigee $\omega_{0}$ to a target orbit with eccentricity $e_{f}$, semimajor axis $a_{f}$ ' and argument of perigee $\omega_{f}$. The initial time is specified to be $t_{0}$, and the final time is specified to be $t_{f}$. The location of the spacecraft in the initial and final orbit is free because the initial and final true anomalies are free variables. The constraints requiring the spacecraft transfer between the initial and final orbit are specified by (61) and (62). The initial covariance is defined to be $\mathbf{P}_{0}$, and the spectral density of the uncertainty in the dynamic equations is given by the constant matrix $\mathbf{Q}$. The measurements are continuous scalar range measurements from a single observer to the spacecraft. The observer's location is specified by $\mathbf{p}(t)$, which is a vector from the center of the central body to the observer. The spectral density of the uncertainty in the measurement is given by the constant matrix $\mathbf{R}$, which is a scalar. By utilizing these conditions (5) to (10) and (14) can now be written as (63) to (66). The specific cost function for the examples in this paper is given by (67), where $q$ is the integral of the trace of the covariance. 


$$
\begin{aligned}
& \mathbf{g}(\mathbf{r})=-\mu \frac{\mathbf{r}}{r^{3}} \\
& \theta=\left(\begin{array}{c}
\mathbf{r}\left(t_{0}\right)-\frac{a_{0}\left(1-e_{0}^{2}\right)}{1+e_{0} \cos \left(\kappa_{0}\right)}\left(\begin{array}{c}
\cos \left(\kappa_{0}+\omega_{0}\right) \\
\sin \left(\kappa_{0}+\omega_{0}\right)
\end{array}\right) \\
\mathbf{v}\left(t_{0}\right)+\sqrt{\frac{\mu}{\left(1-e_{0}^{2}\right)}\left(\begin{array}{c}
\sin \left(\kappa_{0}+\omega_{0}\right)+e_{0} \sin \left(\omega_{0}\right) \\
-\cos \left(\kappa_{0}+\omega_{0}\right)-\cos \left(\omega_{0}\right)
\end{array}\right)} \\
t_{0}-t_{0 s}
\end{array}\right) \\
& \psi=\left(\begin{array}{c}
\mathbf{r}\left(t_{f}\right)-\frac{a_{f}\left(1-e_{f}^{2}\right)}{1+e_{f} \cos \left(\kappa_{f}\right)}\left(\begin{array}{c}
\cos \left(\kappa_{f}+\omega_{f}\right) \\
\sin \left(\kappa_{f}+\omega_{f}\right)
\end{array}\right) \\
\mathbf{v}\left(t_{f}\right)+\sqrt{\frac{\mu}{\left(1-e_{f}^{2}\right)}\left(\begin{array}{c}
\sin \left(\kappa_{f}+\omega_{f}\right)+e_{0} \sin \left(\omega_{f}\right) \\
-\cos \left(\kappa_{f}+\omega_{f}\right)-\cos \left(\omega_{f}\right)
\end{array}\right)} \\
t_{f}-t_{f s}
\end{array}\right) \\
& \left(\begin{array}{c}
\mathbf{r}(t) \\
\mathbf{v}(t)
\end{array}\right)=\left(\begin{array}{cc}
0 & \mathbf{I} \\
\frac{\partial \mathbf{g}}{\partial \mathbf{r}} & 0
\end{array}\right)\left(\begin{array}{l}
\mathbf{r} \\
\mathbf{v}
\end{array}\right)+\left(\begin{array}{c}
0 \\
0 \\
w_{x} \\
w_{y}
\end{array}\right) \\
& \mathbf{z}(t)=\left(\frac{\mathbf{r}_{s}^{T}}{r_{s}} 0^{T}\right)\left(\begin{array}{l}
\mathbf{r}(t) \\
\mathbf{v}(t)
\end{array}\right)+n_{1} \\
& \tilde{\mathbf{X}}^{T} \quad=\left(\mathbf{r}^{T} \mathbf{v}^{T} J P_{1} P_{2} P_{3} P_{4} P_{5} P_{6} P_{7} P_{8} P_{9} P_{10} q\right) \\
& \lambda^{T}=\left(\lambda_{\mathbf{r}}^{T} \lambda_{\mathbf{v}}^{T} \lambda_{J} \lambda_{1} \lambda_{2} \lambda_{3} \lambda_{4} \lambda_{5} \lambda_{6} \lambda_{7} \lambda_{8} \lambda_{9} \lambda_{10} \lambda_{q}\right) \\
& W=\varphi_{1} P_{x x}\left(t_{f}\right)+\varphi_{2} P_{y y}\left(t_{f}\right)+\varphi_{3} P_{x x}\left(t_{f}\right) \\
& +\varphi_{4} P_{y y}\left(t_{f}\right)+\varphi_{5} J\left(t_{f}\right)+\varphi_{6} q\left(t_{f}\right) \text {. } \\
& (60)(61)(62)(63)(64)(65)(66)(67)
\end{aligned}
$$

The six scalars in the cost function, denoted by $\varphi$, determine the relative importance of each element of the trace of the covariance at the final time, the integral of the trace of the covariance, and the propellant. The mass optimal solution is obtained by setting all of

IEEE Transactions on Aerospace and Electronic Systems, Vol 46, No. 2 (April 2010): 771-791. DOI. This article is (C) Institute of Electrical and Electronics Engineers (IEEE) and permission has been granted for this version to appear in e-Publications@Marquette. Institute of Electrical and Electronics Engineers (IEEE) does not grant permission for this article to be further copied/distributed or hosted elsewhere without the express permission from Institute of Electrical and Electronics Engineers (IEEE). 
the $\varphi_{j}$ to zero except for $\varphi_{5}$. In order to place more emphasis on the trajectory's observability at the final time, $\varphi_{1}$ to $\varphi_{4}$ can be increased. Increasing $\varphi_{6}$ places more emphasis on minimizing the trace of the covariance along the entire trajectory. Because the magnitudes of the $\varphi_{i}$ are irrelevant and because only the relative values of the $\varphi_{i}$ are important, $\varphi_{5}$ is always set to one. If all the scalars are positive, the goal is to minimize the cost function; if the scalars are negative, the goal is to maximize the cost function.

The problem can now be solved as a TPBVP, with the 18 unknowns given by (68). The combination of $\alpha$ and (61) specifies the initial states and costates of the system given by (65) and (66). The initial position and velocity costates are determined using the adjoint control transformation. ${ }^{22,23}$ By utilizing (15) and (28), the time derivative of the costates along the optimal path can be written. The explicit expression is omitted here because of its length. The inclusion of (29) and (30) specifies the equations of motion for the entire system (states and costates).

$$
\begin{aligned}
\boldsymbol{\alpha}^{T}= & \left(\lambda_{\mathbf{r}}^{T}\left(t_{0}\right) \lambda_{\mathbf{v}}^{T}\left(t_{0}\right) \lambda_{J}\left(t_{0}\right) \lambda_{1}\left(t_{0}\right)\right. \\
& \left.\ldots \lambda_{10}\left(t_{0}\right) \lambda_{q} \kappa_{0} \kappa_{f}\right) .
\end{aligned}
$$

The constraints that form the TPBVP are determined from (23), (26), and (62). Equation (23) provides the 12 constraints given by (69), and (62) provides four additional constraints. The final two constraints given by (70) are provided by (23) and (26). Any trajectory satisfying these equations is first-order extremal.

IEEE Transactions on Aerospace and Electronic Systems, Vol 46, No. 2 (April 2010): 771-791. DOI. This article is @ Institute of Electrical and Electronics Engineers (IEEE) and permission has been granted for this version to appear in e-Publications@Marquette. Institute of Electrical and Electronics Engineers (IEEE) does not grant permission for this article to be further copied/distributed or hosted elsewhere without the express permission from Institute of Electrical and Electronics Engineers (IEEE). 


$$
\begin{aligned}
\lambda_{J}\left(t_{f}\right) & =\varphi_{5} \\
\lambda_{1}\left(t_{f}\right) & =\varphi_{1} \\
\lambda_{5}\left(t_{f}\right) & =\varphi_{2} \\
\lambda_{8}\left(t_{f}\right) & =\varphi_{3} \\
\lambda_{10}\left(t_{f}\right) & =\varphi_{4} \\
\lambda_{q}\left(t_{f}\right) & =\varphi_{6} \\
\lambda_{2}\left(t_{f}\right) & =\lambda_{3}\left(t_{f}\right)=\lambda_{4}\left(t_{f}\right)(69)(70) \\
\lambda_{\mathbf{r}}^{T}\left(t_{0}\right) \frac{\partial \mathbf{r}\left(t_{0}\right)}{\partial \kappa_{0}}+\lambda_{\mathbf{v}}^{T}\left(t_{0}\right) \frac{\partial \mathbf{v}\left(t_{0}\right)}{\partial \kappa_{0}} & =0 \\
\lambda_{\mathbf{r}}^{T}\left(t_{f}\right) \frac{\partial \mathbf{r}\left(t_{f}\right)}{\partial \kappa_{f}}+\lambda_{\mathbf{v}}^{T}\left(t_{f}\right) \frac{\partial \mathbf{v}\left(t_{f}\right)}{\partial \kappa_{f}} & =0 .
\end{aligned}
$$

\section{B. Continuous Scalar Range Measurements}

The example used to illustrate this technique is a 20,000 second-planar transfer from an initial low Earth orbit (LEO) with eccentricity equal to 0.05 , semimajor axis equal to $7,000 \mathrm{~km}$, and argument of perigee equal to zero to a near GEO with eccentricity equal to 0.1 , semimajor axis equal to $42,200 \mathrm{~km}$, and argument of perigee equal to zero. Unless specified all distance units are kilometers, and all time units are seconds. The Earth is the central gravity body, and all third-body effects are ignored. The observer is located at the center of the Earth in this example. In all of the examples that follow, $\varphi_{1}, \varphi_{2}, \varphi_{3}$, and $\varphi_{4}$ always have the same value, defined to be $\ell$. Unless specified the spectral density matrices for all the examples and the initial value of the covariance are given by (71) to (73). These values are determined based on the sensors on the spacecraft and based the models of the equations of motion that are employed. Additionally $q(t)$ is always the integral of the equally weighted sum of the diagonal elements of the covariance matrix. Because these problems are solved with distance units of kilometers and time units of seconds, the magnitudes of the covariance elements associated with position are orders of magnitude larger than the magnitudes of the covariance elements associated with velocity. In effect this choice of scaling places a much greater emphasis on decreasing $P_{1}$ and $P_{5}$ than on decreasing $P_{8}$ and $P_{10}$. To emphasize all elements of the covariance matrix equally, one 
could easily choose $\varphi_{3}$ and $\varphi_{4}$ to be orders of magnitude larger than $\varphi_{1}$ and $\varphi_{2}$ and could determine $q(t)$ from (74) instead of as the trace of the covariance.

$$
\begin{aligned}
& \mathbf{P}\left(t_{0}\right) \quad=\left(\begin{array}{cccc}
10^{-5} & 0 & 0 & 0 \\
0 & 10^{-5} & 0 & 0 \\
0 & 0 & 10^{-8} & 0 \\
0 & 0 & 0 & 10^{-8}
\end{array}\right) \\
& \mathbf{Q} \quad=\left(\begin{array}{cc}
w_{1}^{2} & 0 \\
0 & w_{1}^{2}
\end{array}\right)=\left(\begin{array}{cc}
10^{-16} & 0 \\
0 & 10^{-16}
\end{array}\right) \\
& \text { R }=10^{-1} \\
& q(t)=\int_{t_{0}}^{t}\left(P_{x x}+P_{y y}+10^{8} P_{x x}^{\ldots}+10^{8} P_{y y}^{\ldots}\right) d t .
\end{aligned}
$$

$(71)(72)(73)(74)$

In Figs. 1-7 $\varphi$ is zero, and the value of $\varphi_{6}$ is increased in order to decrease the integral of the trace of the spacecraft's covariance. In Fig. 1 it is clear that the paths followed on the optimal trajectories obtained with the integral of trace of the covariance increasingly contributing to the cost function are quite similar. In fact at the scale of Fig. 1, the trajectories appear to be the same. In Fig. 2 one can observe that the trajectories that weight the integral of the trace of the covariance more heavily remain closer to the central body during most of the trajectory.
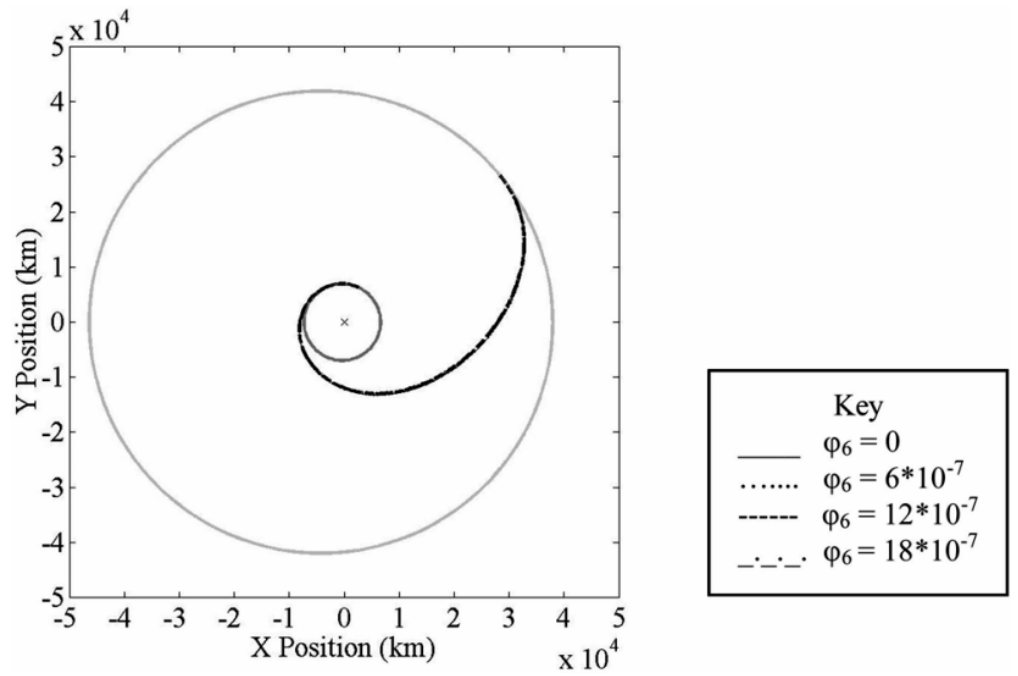

Fig. 1. Optimal trajectories.

IEEE Transactions on Aerospace and Electronic Systems, Vol 46, No. 2 (April 2010): 771-791. DOI. This article is @ Institute of Electrical and Electronics Engineers (IEEE) and permission has been granted for this version to appear in e-Publications@Marquette. Institute of Electrical and Electronics Engineers (IEEE) does not grant permission for this article to be further copied/distributed or hosted elsewhere without the express permission from Institute of Electrical and Electronics Engineers (IEEE). 
NOT THE PUBLISHED VERSION; this is the author's final, peer-reviewed manuscript. The published version may be accessed by following the link in the citation at the bottom of the page.

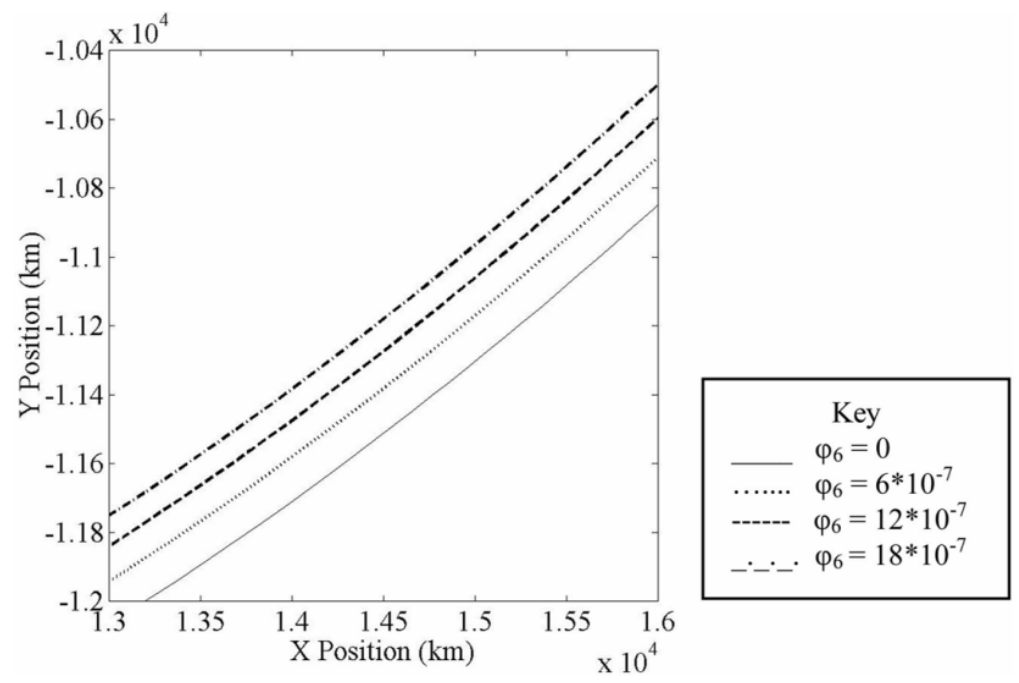

Fig. 2. Magnified portion of optimal trajectories.
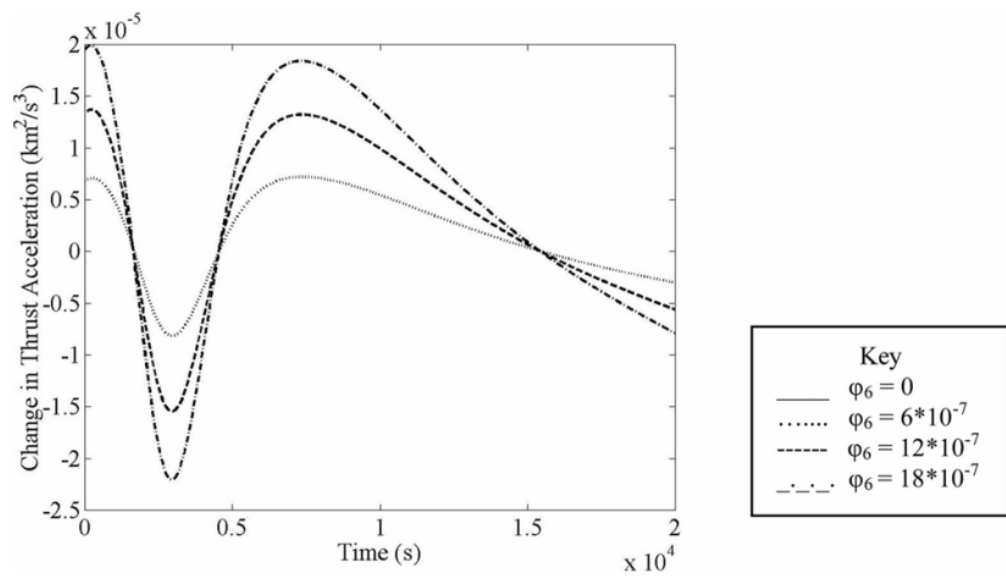

Fig. 3. Perturbation to thrust acceleration magnitude caused by including observability in cost function.
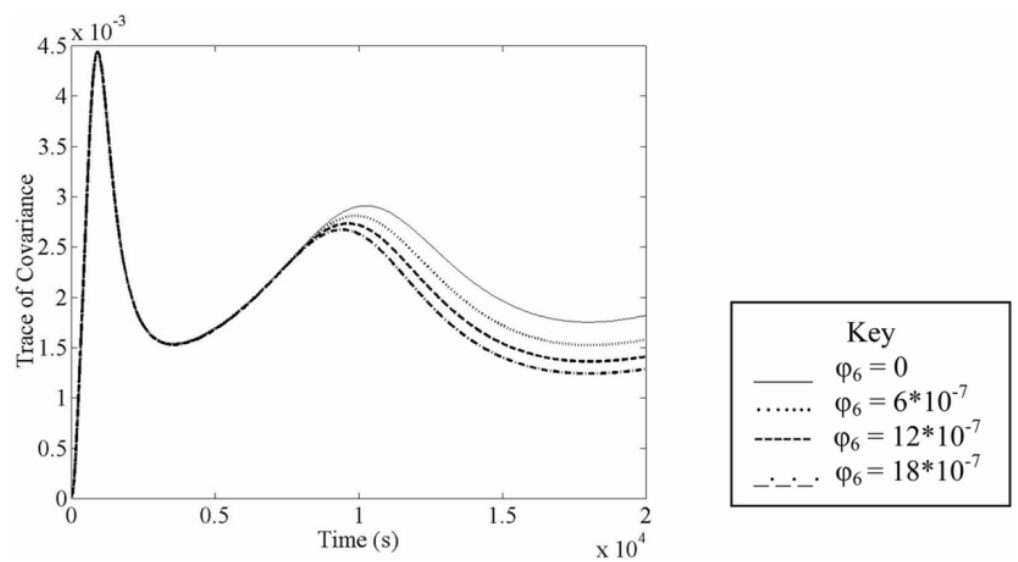

Fig. 4. Trace of covariance versus time.

IEEE Transactions on Aerospace and Electronic Systems, Vol 46, No. 2 (April 2010): 771-791. DOI. This article is (C Institute of Electrical and Electronics Engineers (IEEE) and permission has been granted for this version to appear in e-Publications@Marquette. Institute of Electrical and Electronics Engineers (IEEE) does not grant permission for this article to be further copied/distributed or hosted elsewhere without the express permission from Institute of Electrical and Electronics Engineers (IEEE). 
NOT THE PUBLISHED VERSION; this is the author's final, peer-reviewed manuscript. The published version may be accessed by following the link in the citation at the bottom of the page.

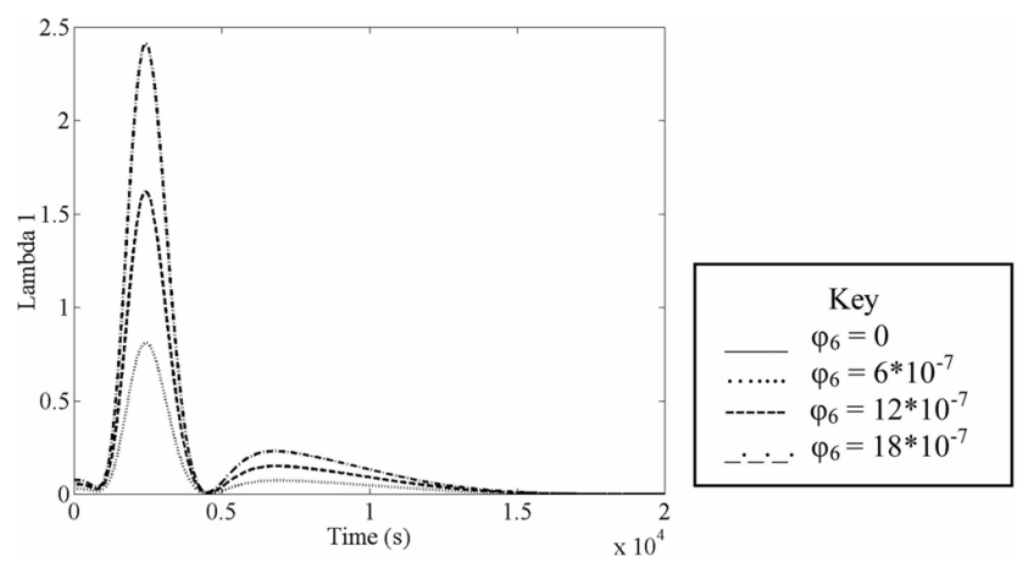

Fig. 5. $\lambda 1$ versus time.

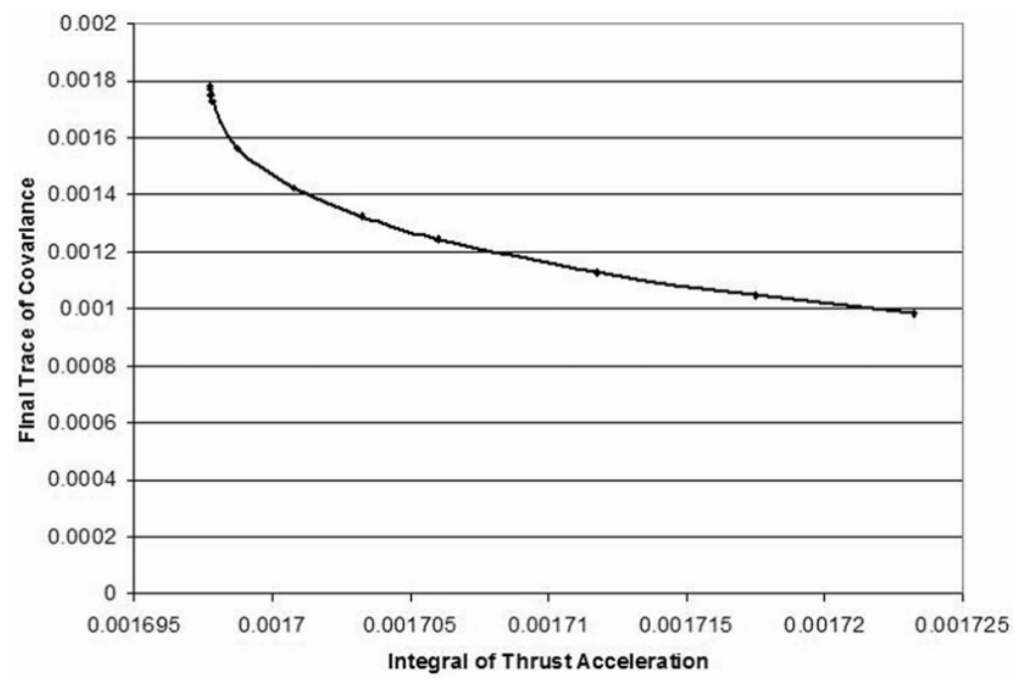

Fig. 6. Pareto front for optimal solutions.

IEEE Transactions on Aerospace and Electronic Systems, Vol 46, No. 2 (April 2010): 771-791. DOI. This article is (C) Institute of Electrical and Electronics Engineers (IEEE) and permission has been granted for this version to appear in e-Publications@Marquette. Institute of Electrical and Electronics Engineers (IEEE) does not grant permission for this article to be further copied/distributed or hosted elsewhere without the express permission from Institute of Electrical and Electronics Engineers (IEEE). 
NOT THE PUBLISHED VERSION; this is the author's final, peer-reviewed manuscript. The published version may be accessed by following the link in the citation at the bottom of the page.

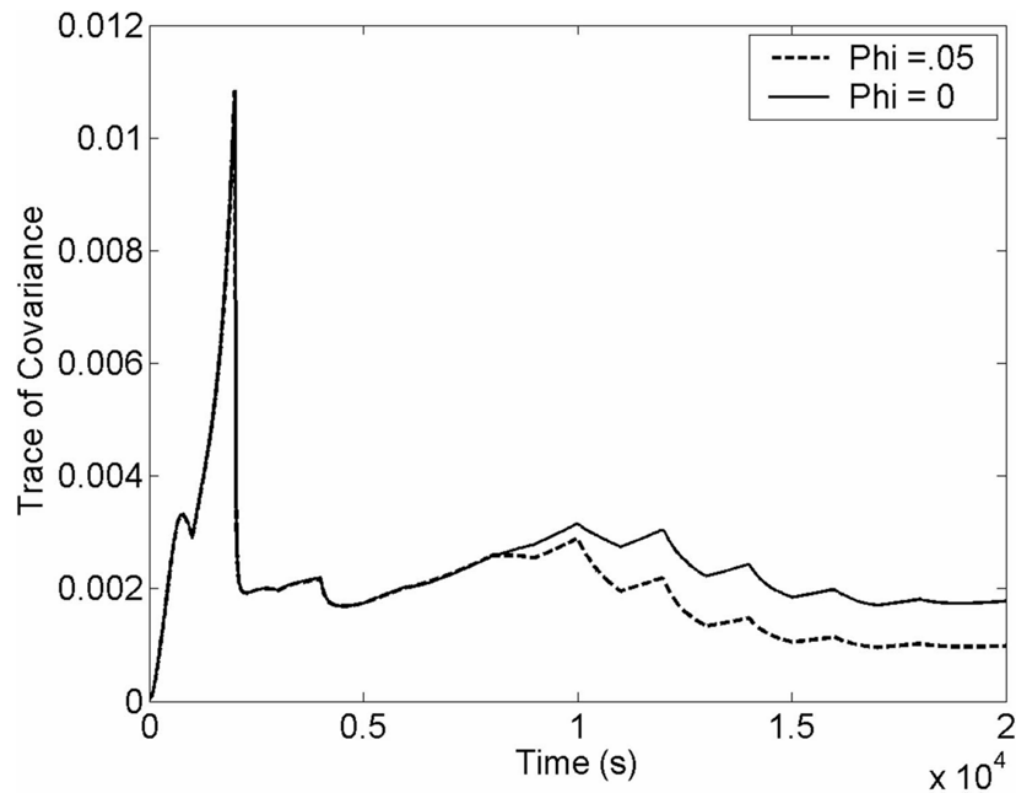

Fig. 7. Trace of covariance versus time.

Fig. 3 shows the difference between the thrust acceleration magnitude on trajectories that include observability in the cost function and the trajectory that is mass optimal. This difference is on the order of ten percent of the thrust acceleration on the mass optimal transfer.

Fig. 4 shows the major difference between the optimal trajectories that incorporate covariance into the cost function. As the value of $\varphi_{6}$ is increased, the trace of the covariance is decreased significantly. Table I illustrates that a small increase in fuel consumption can significantly reduce the covariance. Between the mass optimal solution and the solution with $\varphi_{6}$ equal to $18 * 10^{-7}$, the integral of the trace of the covariance is reduced by over 12 percent, while the integral of the thrust acceleration squared is only increased by about one-half of one percent. Notice the diminishing returns that are obtained by increasing the value of $\varphi_{6}$. As $\varphi_{6}$ is increased from 0 to $6 * 10^{-7}$, it takes less additional fuel to reduce the integral of the trace of the covariance than it does as $\varphi_{6}$ is increased from 6 to $12 * 10^{-7}$.

Table I Observability and Fuel Usage for Trajectories Minimizing the Integral of the Trace of the Covariance

\begin{tabular}{|c|c|c|c|c|c|c|}
\hline$\varphi_{6} * 10^{-7}$ & $\begin{array}{l}\text { Integral of Thrust } \\
\text { Acc. Squared } * 10^{-4}\end{array}$ & $\begin{array}{l}\text { Change in Integral } \\
\text { of Thrust Acc. } \\
\text { Squared }+10^{-7}\end{array}$ & $\begin{array}{c}\text { Integral of Trace } \\
\text { of Cov. }\end{array}$ & $\begin{array}{l}\text { Change in Integral } \\
\text { of Trace of Cov. }\end{array}$ & $\begin{array}{l}\text { Trace of Cov, at } \\
\text { Final Time } * 10^{-3}\end{array}$ & $\begin{array}{c}\text { Change in Final } \\
\text { Trace of Cov. } \\
+10^{-4}\end{array}$ \\
\hline 0 & 16.9774 & N/A & 43.7312 & N/A & 1.8217 & N/A \\
\hline 6 & 16.9910 & 13.6 & 41.3646 & -2.3666 & 1.5806 & -2.411 \\
\hline 12 & 17.0230 & 45.6 & 39.5520 & -4.1792 & 1.4122 & -4.095 \\
\hline 18 & 17,0662 & 88.8 & 38.1030 & -5.6282 & 1.2876 & -5.341 \\
\hline
\end{tabular}

IEEE Transactions on Aerospace and Electronic Systems, Vol 46, No. 2 (April 2010): 771-791. DOI. This article is @ Institute of Electrical and Electronics Engineers (IEEE) and permission has been granted for this version to appear in e-Publications@Marquette. Institute of Electrical and Electronics Engineers (IEEE) does not grant permission for this article to be further copied/distributed or hosted elsewhere without the express permission from Institute of Electrical and Electronics Engineers (IEEE). 
Fig. 5 shows a sampling of the behavior of the costates associated with the elements of the covariance matrix. As the value of $\varphi_{6}$ is increased, the graphs of the costate retain the same shape with the amplitude increasing. This observation is true for all of the costates, not just the sample illustrated in this figure.

In the previous example $\varphi$ was set to zero, and the value of $\varphi_{6}$ was increased in order to incorporate observability into the optimization process. Alternatively one could set $\varphi_{6}$ to zero and increase the value of $\varphi$ in order to minimize the trace of the covariance at the final time instead of the integral of the trace of the covariance. This technique provides similar results to the above example. In fact plots of the trajectories, controls, and costates are similar to those shown above, with one exception. As $\varphi$ is increased the trajectories no longer remain closer to the central body during the majority of the trajectory as they do in the examples where $\varphi_{6}$ is increased. In fact there is no correlation between the distance the spacecraft is from the central body during the majority of the trajectory and the value of $\varphi$.

Table II provides a summary of the fuel consumption and observability along each trajectory. The trace of the covariance at the final time can be reduced by over 31 percent with less than a 0.6 percent increase in the integral of the thrust acceleration squared. for this specific transfer problem, either method (increasing $\varphi$ or increasing $\varphi_{6}$ ) results in a trajectory where both the integral of the trace of the covariance and the trace of the covariance at the final time are reduced. As expected increasing $\varphi$ more efficiently reduces the trace of the covariance at the final time, while increasing $\varphi_{6}$ allows the integral of the trace of the covariance to be reduced with a smaller fuel penalty. In other transfer problems increasing $\varphi_{6}$ may cause the trace of the covariance to be greater at the final time, even though it reduces the integral of the trace of the covariance. Similarly increasing $\varphi$ may cause the integral of the trace of the covariance to increase, even though it reduces the trace of the covariance at the final time. The values $\varphi$ and $\varphi_{6}$ that produce the optimal trajectory (trajectory that balances observability and fuel efficiency optimally for a particular mission) depend on the mission. 
NOT THE PUBLISHED VERSION; this is the author's final, peer-reviewed manuscript. The published version may be accessed by following the link in the citation at the bottom of the page.

Table II Observability and Fuel Usage for Trajectories Minimizing the Trace of the Covariance at the Final Time

\begin{tabular}{|c|c|c|c|c|c|c|}
\hline$\varphi=10^{-4}$ & $\begin{array}{l}\text { Integral of Thrust } \\
\text { Acc. Squared } * 10^{-4}\end{array}$ & $\begin{array}{c}\text { Change in Integral } \\
\text { of Thrust Acc, } \\
\text { Squared } * 10^{-7}\end{array}$ & $\begin{array}{c}\text { Integral of Trace } \\
\text { of Cov. }\end{array}$ & $\begin{array}{l}\text { Change in Integral } \\
\text { of Trace of Cov. }\end{array}$ & $\begin{array}{l}\text { Trace of Cov, at } \\
\text { Final Time } * 10^{-3}\end{array}$ & $\begin{array}{l}\text { Change in Final } \\
\text { Trace of Cov. } \\
+10^{-4}\end{array}$ \\
\hline 0 & 16.977 & $\mathrm{~N} / \mathrm{A}$ & 43.7312 & $\mathrm{~N} / \mathrm{A}$ & 1.8217 & N/A \\
\hline 55 & 16.989 & 11.6 & 41.5930 & -2.1382 & 1.5894 & -2.323 \\
\hline 110 & 17.013 & 35.6 & 40.1272 & -3.6040 & 1.4411 & -3.806 \\
\hline 165 & 17.042 & 64.6 & 39.0203 & -4.7109 & 1.3348 & -4.869 \\
\hline 200 & 17.062 & 84.2 & 38.4371 & -5.2941 & 1.2807 & -5.410 \\
\hline 220 & 17.073 & 95.6 & 38.1359 & -5.5953 & 1.2533 & -5.6842 \\
\hline
\end{tabular}

\section{Periods without Measurements}

The optimality conditions derived from using the calculus of variations for the case of continuous observations throughout the transfer are largely unchanged if there are periods when no observations are taken. The times when observations begin and end are not optimized and must be determined a priori. The time derivative of the covariance matrix instantaneously changes between (11) and (12), when observations begin and end. Consequently the Hamiltonian defined by (75) changes as well. Because the optimality conditions require the time derivative of the costates be given by (76), the time derivative of the costates changes as well. Both the augmented state and costate vectors are continuous at the times when observations start or stop. The other optimality conditions determined by the calculus of variations are unchanged.

$(75)(76)$

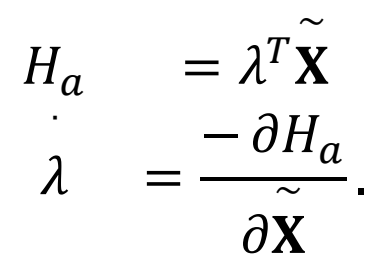

The same transfer as the first example, with all of the parameters unchanged except for the spectral density associated with the measurement, is used to illustrate the changes necessary when there are periods without observations. Continuous scalar range measurements are taken when the thousands digit of the elapsed time is an even number. Because the measurements are only taken half of the time, the spectral density associated with the measurement error is reduced by one-half, as specified in (77). This value of the spectral density causes the final value of the trace of the covariance on the mass optimal solution to be nearly the same in this example and the first example. 


$$
\boldsymbol{R}=0.05
$$

The results from optimizing the trajectory with periods that have no measurements are similar to the results obtained in the first example, when observations were made continuously throughout the trajectory. Table III provides a summary of the fuel cost and trace of the covariance at the final time for various values of $\varphi$. Notice that $\varphi_{6}$ is zero in this example. The trace of the covariance can be reduced by 30 percent for about a one-half percent increase in the integral of the thrust acceleration squared. The results indicate that the fuel cost of reducing the covariance at the final time is the same whether the measurements are continuous throughout the transfer or there are periods without measurements. The diminishing returns can be clearly seen in the Pareto front in Fig. 6. Figs. 7 and 8 show the trace of the covariance and costates for various values of $\varphi$. These figures, as well as the optimal trajectory and control profile, are remarkably similar to the figures for the same variables for the case of continuous measurements. The costates follow a curve with the same shape and phase behavior as the results from the first example.

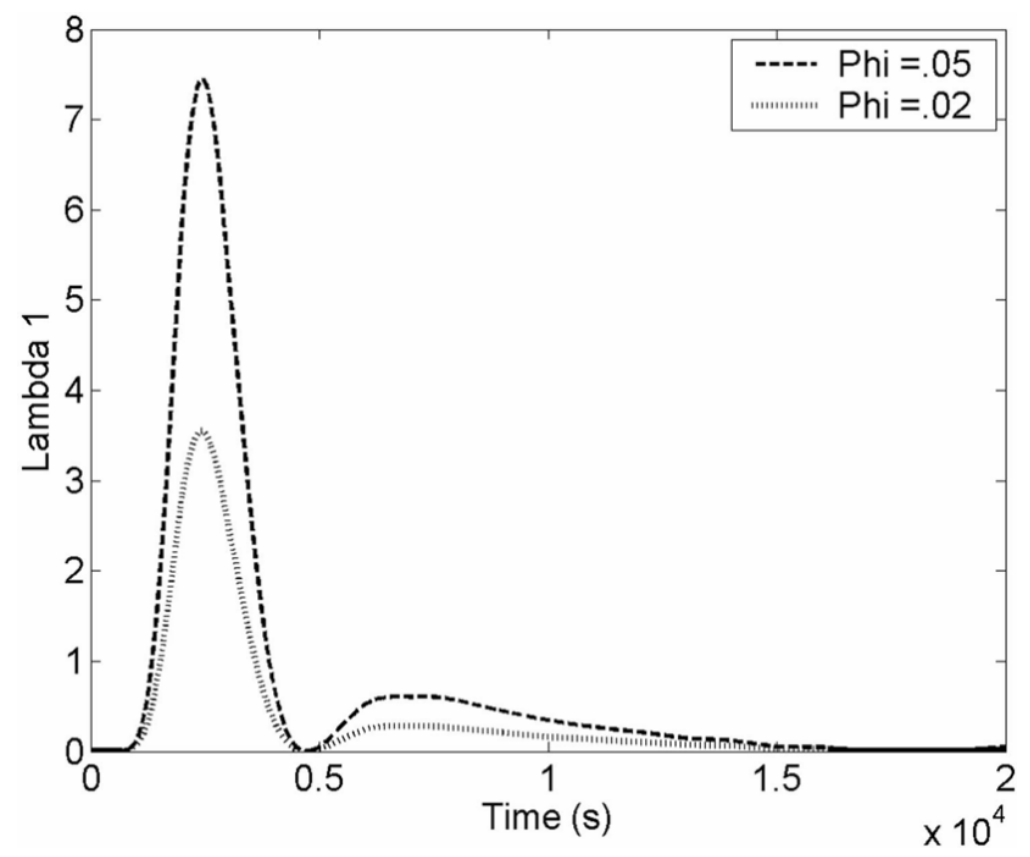

Fig. 8. $\lambda 1$ versus time. 
Table III Integral of Thrust Acceleration Squared and Trace of Covariance at Final Time with Periods of No Observations

\begin{tabular}{|c|c|c|c|c|c|}
\hline$\varphi$ & $\begin{array}{l}\text { Integral of Thrust } \\
\text { Squared } * 10^{-4}\end{array}$ & $\begin{array}{l}\text { Change in Integral } \\
\text { of Thrust Acc, } \\
\text { Squared } * 10^{-10}\end{array}$ & $\begin{array}{l}\text { Trace of Cov, at } \\
\text { Final Time } * 10^{-3}\end{array}$ & $\begin{array}{l}\text { Change in Final } \\
\text { Trace of Cov. } * 10^{-6}\end{array}$ & $\frac{\Delta \operatorname{Tr}[\mathbf{P}]}{\Delta J}$ \\
\hline 0 & 16.97740 & $\mathrm{~N} / \mathrm{A}$ & 1.77833 & $\mathrm{~N} / \mathrm{A}$ & N/A \\
\hline .0001 & 16.97741 & 6 & 1.77285 & -5.5 & 913.35 \\
\hline .0005 & 16.97753 & 134 & 1.75157 & -26.8 & 166.25 \\
\hline .0010 & 16.97791 & 510 & 1.72632 & -52.0 & 67.15 \\
\hline .0050 & 16.98720 & 9804 & 1.56459 & -213.7 & 17.4 \\
\hline .0100 & 17.00772 & 30320 & 1.42504 & -353.3 & 6.8 \\
\hline .0150 & 17.03281 & 55408 & 1.32372 & -454.6 & 4.05 \\
\hline .0200 & 17.06007 & 82674 & 1.24538 & -533.0 & 2.85 \\
\hline .0300 & 17.11718 & 139784 & 1.12974 & -648.6 & 2 \\
\hline .0400 & 17.17500 & 197600 & 1.04657 & -731.8 & 1.45 \\
\hline .0500 & 17.23222 & 254816 & 0.98271 & -795.6 & 1.1 \\
\hline
\end{tabular}

\section{Discrete Scalar Range Measurements}

The same transfer as the previous two examples is used to illustrate the discrete measurements case. The covariance associated with the noise in the measurement, given by (78), is different than the spectral density specified in (73) because the covariance term is not integrated over the time between each measurement. The value of the covariance associated with the noise in the measurement is selected so that the final value of the covariance for the mass optimal case is of the same order of magnitude as the mass optimal solution from the first example that uses continuous measurements. A discrete scalar range measurement from the center of the Earth to the spacecraft is taken every 400 seconds, beginning at $t=400$ and ending at $t=19,600$.

$$
\tilde{\mathbf{R}}=.00025
$$

To illustrate the constraints specified by $\sigma$, (79), where $s$ is defined in (80), gives the third unique constraint from (13). The expression represented by $s$ is a scalar because the measurements can always be processed one at a time. The value of one element of the $\mathrm{L}$ matrix is given by (81). The other elements of $\sigma$ and $\mathrm{L}$ can be determined from (13) and (39). When the observations are scalar range measurements, the determinant of B is given by (82), which can never be zero as long as there is noise in the measurement and as long as the observer never occupies the same location as the spacecraft. As a result one can solve (38) to determine the unique costates after each discrete measurement. 


$$
\begin{aligned}
\sigma_{3} & =P_{x x} \cdot\left(t_{k+}\right)-P_{x x} \cdot\left(t_{k-}\right)+\frac{s}{x_{s}^{2}+y_{s}^{2}} \\
& \times\left[P_{x x}\left(x_{s}^{2} P_{x x}+x_{s} y_{s} P_{y x}\right)+P_{x y}\left(x_{s} y_{s} P_{x x}+y_{s}^{2} P_{y x}\right)\right] I_{t_{k-}} \\
s & =\left(\mathbf{H}\left(t_{k}\right) \mathbf{P}\left(t_{k-}\right) \mathbf{H}^{T}\left(t_{k}\right)+\mathbf{R}\right)^{-1} \\
L(6,3) & =\left.\frac{s}{x_{s}^{2}+y_{s}^{2}}\left[P_{x x} x_{s} y_{s}+P_{x y} y_{s}^{2}\right]\right|_{t_{k-}} \\
|\mathbf{B}| & =\frac{\tilde{R}\left(x_{s}^{2}+y_{s}^{2}\right)}{P_{x x} x_{s}^{2}+P_{x y} x_{s} y_{s}+P_{y y} y_{s}^{2}+\tilde{R}\left(x_{s}^{2}+y_{s}^{2}\right)}
\end{aligned}
$$

The results for this discrete measurement example are similar to the results for continuous measurements. The integral of the thrust acceleration squared and the final value of the trace of the Cartesian covariance for the optimal trajectories for various values of $\varphi$ are shown in Table IV. The trace of the covariance at the final time can be reduced by about 20 percent, with an increase of less than two-tenths of one percent in the integral of the thrust acceleration squared. Just as in the case of the continuous measurements, the fuel cost of further reducing the trace of the covariance at the final time increases significantly as $\varphi$ is increased. The trace of the covariance and costates for various values of $\varphi$ are shown in Figs. 9 and 10. Although the plot of the trace of the covariance is discontinuous, the shape of the graph and the time when the covariance on the mass optimal solution diverges from the covariance on the $\varphi=.5$ solution are nearly the same as the previous example with continuous observations. The results are similar for the control and the costates as well. Even though the covariance and costates are discontinuous at each measurement time, the controls still remain continuous at the measurement times. The plots for the other covariance elements and other costates are omitted because they are similar to the plots that are shown. The only elements that are significantly different are the plots for $\lambda_{8}, \lambda_{9}$, and $\lambda_{10}$, which are continuous. 
NOT THE PUBLISHED VERSION; this is the author's final, peer-reviewed manuscript. The published version may be accessed by following the link in the citation at the bottom of the page.

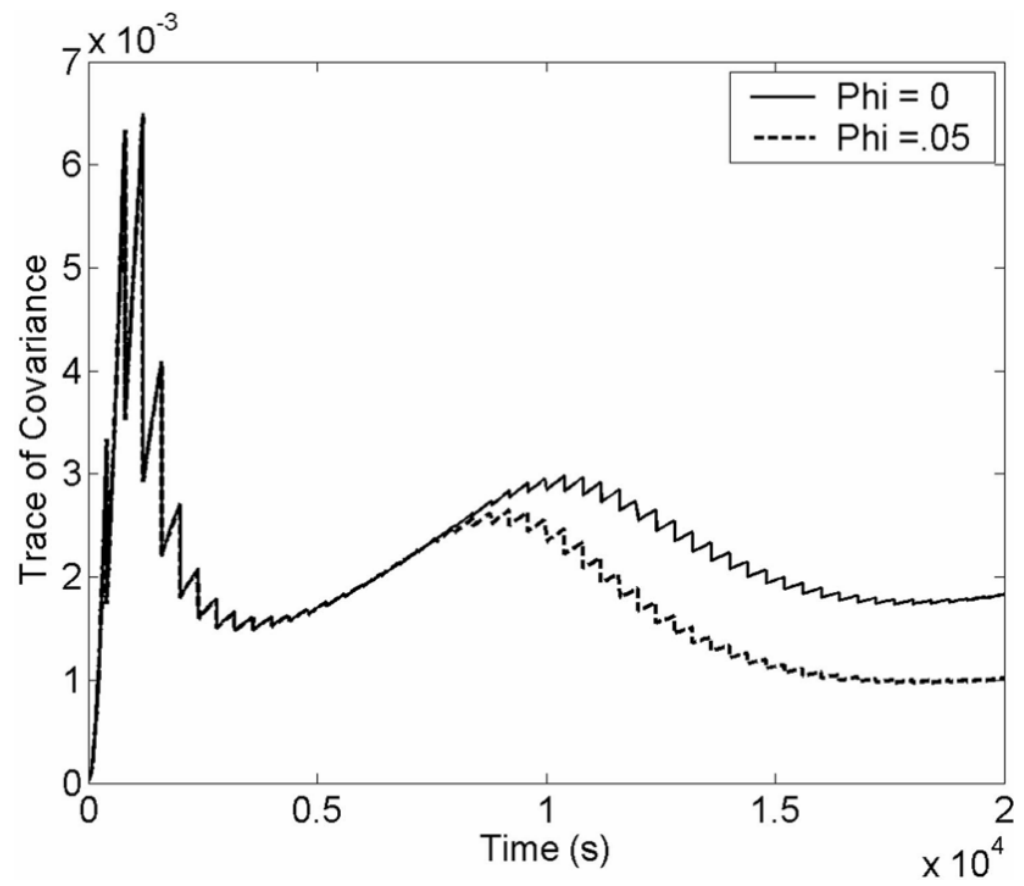

Fig. 9. Trace of covariance versus time.

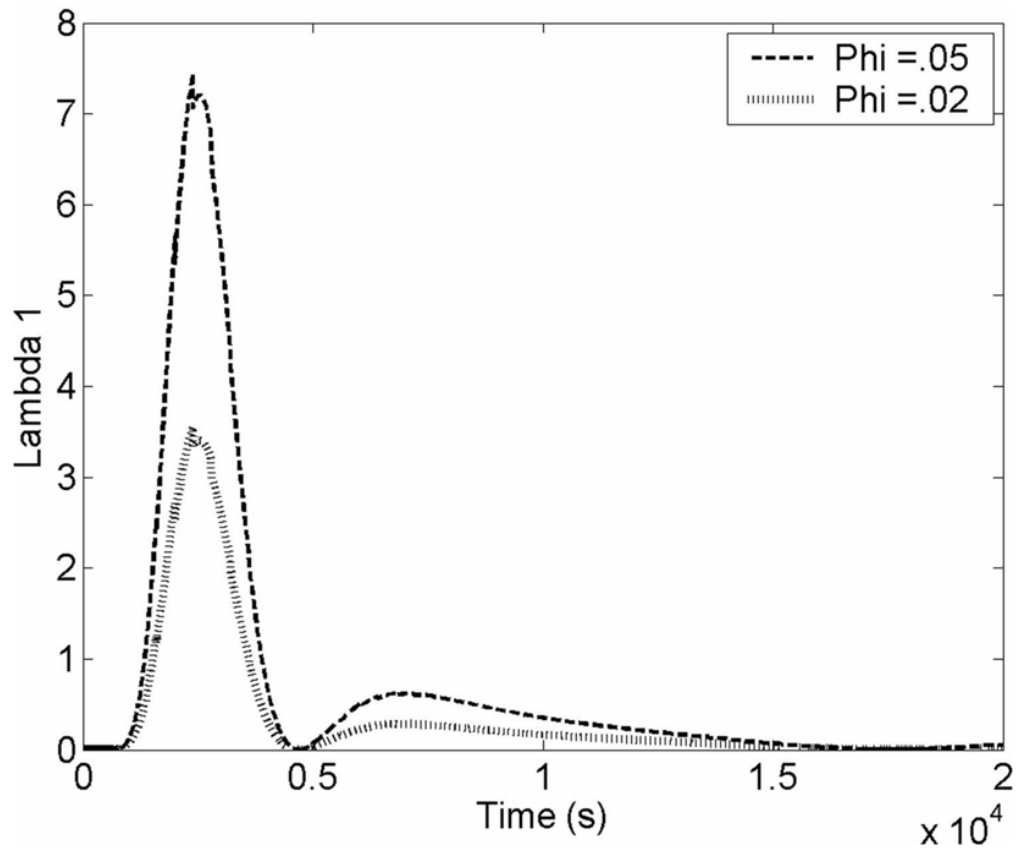

Fig. 10. $\lambda_{1}$ versus time.

IEEE Transactions on Aerospace and Electronic Systems, Vol 46, No. 2 (April 2010): 771-791. DOI. This article is ( Institute of Electrical and Electronics Engineers (IEEE) and permission has been granted for this version to appear in e-Publications@Marquette. Institute of Electrical and Electronics Engineers (IEEE) does not grant permission for this article to be further copied/distributed or hosted elsewhere without the express permission from Institute of Electrical and Electronics Engineers (IEEE). 
Table IV Observability and Fuel Usage for Trajectories Minimizing the Trace of the Covariance at the Final Time with Discrete Observations

\begin{tabular}{|c|c|c|c|c|c|}
\hline$\varphi$ & $\begin{array}{l}\text { Integral of Thrust } \\
\text { Squared } * 10^{-4}\end{array}$ & $\begin{array}{l}\text { Change in Integral } \\
\text { of Thrust Acc. } \\
\text { Squared } * 10^{-10}\end{array}$ & $\begin{array}{l}\text { Trace of Cov, at } \\
\text { Final Time } * 10^{-3}\end{array}$ & $\begin{array}{l}\text { Change in Final } \\
\text { Trace of Cov. } * 10^{-6}\end{array}$ & $\frac{\Delta \operatorname{Tr}[\mathbf{P}]}{\Delta J}$ \\
\hline 0 & 16.97740 & $\mathrm{~N} / \mathrm{A}$ & 1.82928 & $\mathrm{~N} / \mathrm{A}$ & N/A \\
\hline .0001 & 16.97741 & 12 & 1.82355 & -6.1 & 510 \\
\hline .0005 & 16.97754 & 136 & 1.80220 & -27.5 & 172.2 \\
\hline .0010 & 16.97791 & 512 & 1.77683 & -52.8 & 67.45 \\
\hline .0050 & 16.98729 & 9888 & 1.61344 & -216.2 & 17.45 \\
\hline .0100 & 17,00815 & 30752 & 1.47149 & -358.2 & 6.8 \\
\hline .0150 & 17.03384 & 56436 & 1.36794 & -461.7 & 4.05 \\
\hline .0200 & 17.06177 & 84374 & 1.28765 & -542.0 & 2.85 \\
\hline .0300 & 17.12041 & 143010 & 1.16891 & -660.8 & 2.05 \\
\hline .0400 & 17.17986 & 202458 & 1.08339 & -746.3 & 1.45 \\
\hline .0500 & 17.23872 & 261324 & 1.01769 & -812.0 & 1.1 \\
\hline
\end{tabular}

\section{E. Dynamic Errors Based on Thrust}

The same planar transfer from LEO to near GEO is used to illustrate the effect of allowing the error in the dynamic equations to be a function of the controls. The observer is fixed at the center of the Earth taking continuous scalar range measurements of the spacecraft position throughout the trajectory. Because it more accurately reflects the operation of an engine, the second formulation, in which the controls are thrust acceleration magnitude and direction, is used to illustrate this example. The constant term in the spectral density matrix for the error in the dynamic equations $w_{1}^{2}$, given by (72), is reduced to one-half its value in the previous examples. The constants $\xi 1$ and $\xi_{2}$ are set to $1.1 * 10^{8}$ and $10^{8}$, respectively. These values were chosen so that errors in the engine thrust magnitude and direction and errors due to dynamics that were not modeled would be of approximately the same magnitude. $\xi_{1}$ and $\xi_{2}$ have different values in order to make the spectral density matrix $\mathbf{Q}$ nondiagonal.

Table $V$ summarizes the effect that placing an increasing emphasis on the trace of the covariance at the final time has on the required thrust acceleration and the covariance. Again a relatively large decrease in the trace of the covariance at the final time can be obtained for a small increase in the thrust acceleration. As the covariance at the final time is reduced, it becomes more expensive to further reduce the final covariance. Following the trajectory that minimizes the trace of the covariance at the final time has nearly no affect on the trace of the covariance during the initial portion of the transfer, but it does significantly reduce the trace of the covariance at the final time. 
Table V Observability and Fuel Usage for Trajectories Minimizing The Trace of the Covariance at the Final Time with Variable Errors in the Dynamics

\begin{tabular}{ccccccc}
\hline \hline & $\begin{array}{c}\text { Integral of Thrust } \\
\text { Acc. Squared } * 10^{-4}\end{array}$ & $\begin{array}{c}\text { Change in Integral } \\
\text { of Thrust Ace. } \\
\text { Squared } * 10^{-8}\end{array}$ & $\begin{array}{c}\text { Change in Integral } \\
\text { of Cov. }\end{array}$ & $\begin{array}{c}\text { Change in Final } \\
\text { of Trace of Cov. } \\
* 10^{-1}\end{array}$ & $\begin{array}{c}\text { Trace of Cov. } \\
\text { Final Time } * 10^{-3}\end{array}$ & $\begin{array}{c}\text { Trace of Cov. } \\
* 10^{-4}\end{array}$ \\
\hline 0.000 & 16.9774 & N/A & 58.8494 & N/A & 4.1586 & N/A \\
0.002 & 16.9802 & 28 & 57.9147 & -9.347 & 4.0164 & -1.422 \\
0.004 & 16.9878 & 104 & 57.0755 & -17.739 & 3.8897 & -2.689 \\
0.006 & 16.9990 & 216 & 56.3185 & -25.309 & 3.7762 & -3.824 \\
0.008 & 17.0134 & 360 & 55.6321 & -32.173 & 3.6740 & -4.846 \\
0.010 & 17.0300 & 526 & 55.0065 & -38.429 & 3.5815 & -5.771 \\
\hline
\end{tabular}

Table VI shows the effect of placing an increasing emphasis on the integral of the trace of the covariance, instead of the trace of the covariance at the final time. In this case the integral of the trace of the covariance can be reduced by over eight percent, with less than a one-half percent increase in the integral of the thrust acceleration squared. Again the ratio of the percentage decrease in the integral of the trace of the covariance to the percentage increase in the integral of the thrust acceleration squared decreases significantly, as maximizing observability is emphasized. Figs. 11 and 12 illustrate the effect of modeling the error in the dynamic equations as a function of the thrust magnitude. As the integral of the trace of the covariance is weighted more heavily in the optimization process, the maximum value for the thrust acceleration magnitude decreases, thus introducing less uncertainty into the equations of motion.
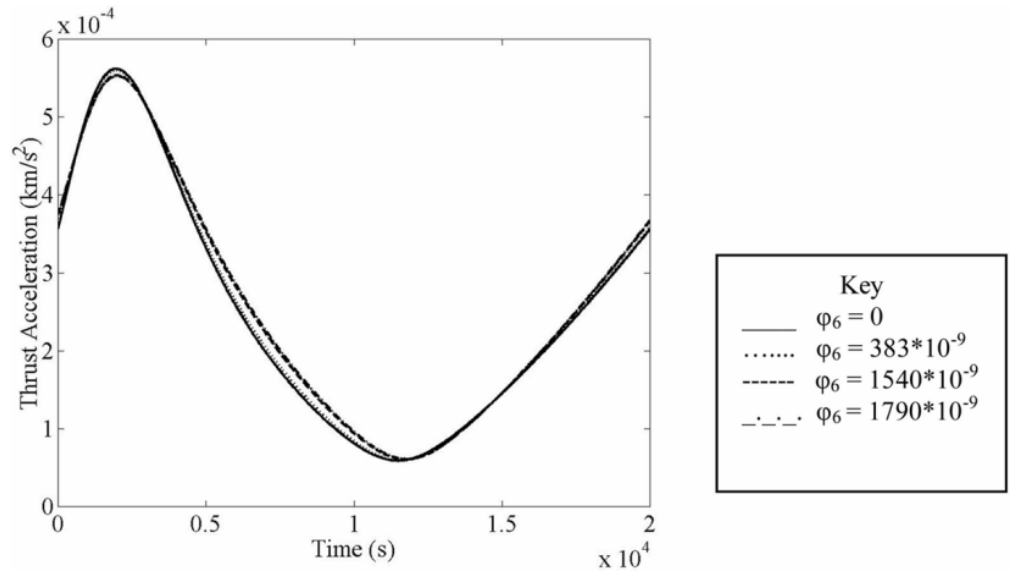

Fig. 11. Thrust acceleration magnitude versus time.

IEEE Transactions on Aerospace and Electronic Systems, Vol 46, No. 2 (April 2010): 771-791. DOI. This article is (C) Institute of Electrical and Electronics Engineers (IEEE) and permission has been granted for this version to appear in e-Publications@Marquette. Institute of Electrical and Electronics Engineers (IEEE) does not grant permission for this article to be further copied/distributed or hosted elsewhere without the express permission from Institute of Electrical and Electronics Engineers (IEEE). 


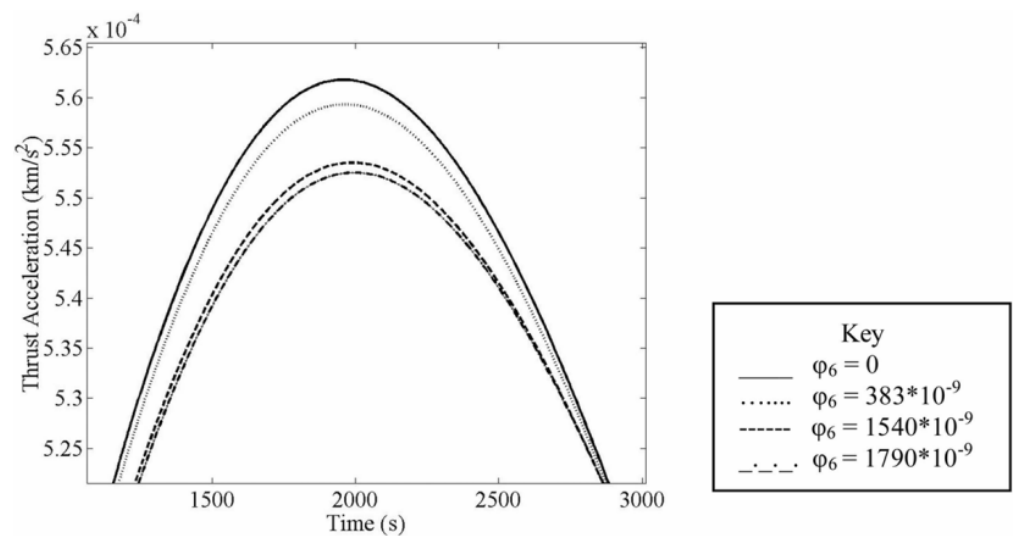

Fig. 12. Maximum thrust acceleration magnitude versus time.

Table VI Observability and Fuel Usage for Trajectories Minimizing the Integral of the Trace of the Covariance with Variable Errors in the Dynamics

\begin{tabular}{rcccccc}
\hline \hline$\varphi_{6} * 10^{-7}$ & $\begin{array}{c}\text { Integral of Thrust } \\
\text { Acc. Squared } * 10^{-4}\end{array}$ & $\begin{array}{c}\text { Change in Integral } \\
\text { of Thrust Ace. } \\
\text { Squared } * 10^{-8}\end{array}$ & $\begin{array}{c}\text { Integral of Trace } \\
\text { of Cov. }\end{array}$ & $\begin{array}{c}\text { Change in Integral } \\
\text { of Trace of Cov. } \\
+10^{-1}\end{array}$ & $\begin{array}{c}\text { Trace of Cov. } \\
\text { Final Time } * 10^{-3}\end{array}$ & $\begin{array}{c}\text { Change in Final } \\
\text { Trace of Cov. } \\
* 10^{-4}\end{array}$ \\
\hline 0 & 16.9774 & N/A & 58.8494 & N/A & 4.159 & N/A \\
3.83 & 16.9822 & 48.4 & 57.5619 & -1.2875 & 3.981 & -1.78 \\
15.4 & 17.0406 & 632.2 & 54.4267 & -4.4227 & 3.563 & -5.96 \\
17.9 & 17.0593 & 819 & 53.8654 & -4.9840 & 3.491 & -6.68 \\
\hline
\end{tabular}

\section{F. Earth Orbit to Lunar Orbit}

A $36 \mathrm{hr}$ planar transfer from an initial Earth orbit, with eccentricity equal to 0.05 , semimajor axis equal to $42,000 \mathrm{~km}$, and argument of perigee equal to zero, to a lunar orbit, with eccentricity equal to 0.1 , semimajor axis equal to $3,476 \mathrm{~km}$ (two Moon radii), and argument of perigee equal to zero, is employed to demonstrate this application. Throughout the trajectory the gravitational effects from both the Earth and the Moon affect the spacecraft. The Moon is modeled as traveling in a circular orbit around the Earth at a distance of $384,400 \mathrm{~km}$. At the beginning of the example transfer, the Moon has a specified mean anomaly, which is set to $190 \mathrm{deg}$. The effect of the initial mean anomaly on the optimal solution can be studied parametrically, or one could add this value to the list of free variables with only slight modifications to the optimality conditions.

During the transfer a series of scalar range measurements of the spacecraft state are taken. The observers are located at three locations on the surface of the Earth, which are separated by $120 \mathrm{deg}$. The locations of these observers are expressed as a function of time in (83). These observations are modeled as alternating periods of continuous measurements and no measurements, with each period lasting for one hour, until the final

IEEE Transactions on Aerospace and Electronic Systems, Vol 46, No. 2 (April 2010): 771-791. DOI. This article is (C) Institute of Electrical and Electronics Engineers (IEEE) and permission has been granted for this version to appear in e-Publications@Marquette. Institute of Electrical and Electronics Engineers (IEEE) does not grant permission for this article to be further copied/distributed or hosted elsewhere without the express permission from Institute of Electrical and Electronics Engineers (IEEE). 
two hours of the transfer, during which continuous observations are made. The measurements are taken by the observer who views the spacecraft with the highest elevation at the beginning of the measurement period. Consequently none of the measurements are occulted by the Earth. The cost function is again the sum of the integral of the thrust acceleration squared and the covariance associated with the semimajor axis of the spacecraft, with respect to the Moon at the end of the transfer, as specified by (84).

$$
\begin{aligned}
\mathbf{p}_{1}(t)=6378\left[\begin{array}{l}
\cos \left(\frac{2 \pi t}{86400}\right) \\
\sin \left(\frac{2 \pi t}{86400}\right)
\end{array}\right] \\
\mathbf{p}_{2}(t)=6378\left[\begin{array}{l}
\cos \left(\frac{2 \pi t}{86400}+\frac{2 \pi}{3}\right) \\
\sin \left(\frac{2 \pi t}{86400}+\frac{2 \pi}{3}\right)
\end{array}\right] \\
\mathbf{p}_{3}(t)=6378\left[\begin{array}{l}
\cos \left(\frac{2 \pi t}{86400}+\frac{4 \pi}{3}\right) \\
\sin \left(\frac{2 \pi t}{86400}+\frac{4 \pi}{3}\right)
\end{array}\right] \\
W=J\left(t_{f}\right)+\varphi P_{a a}\left(t_{f}\right)=J\left(t_{f}\right)+\varphi \frac{\partial a}{\mathbf{X}} \mathbf{P}_{\mathbf{x}}\left(t_{f}\right)\left(\frac{\partial a}{\mathbf{X}}\right) .
\end{aligned}
$$

The results from this optimization indicate that the covariance associated with the semimajor axis of the target lunar orbit can be reduced significantly with only a slight increase in thrust acceleration. Table VII documents the integral of the thrust acceleration squared and the covariance associated with the target orbit semimajor axis for several trajectories that increasingly weight the covariance in the cost function. The covariance associated with the semimajor axis of the target orbit can be reduced by over 75 percent, with an increase in the integral of the thrust acceleration squared of only slightly more than three-quarters of one percent. The Pareto front for this solution is shown in Fig. 13. 
NOT THE PUBLISHED VERSION; this is the author's final, peer-reviewed manuscript. The published version may be accessed by following the link in the citation at the bottom of the page.

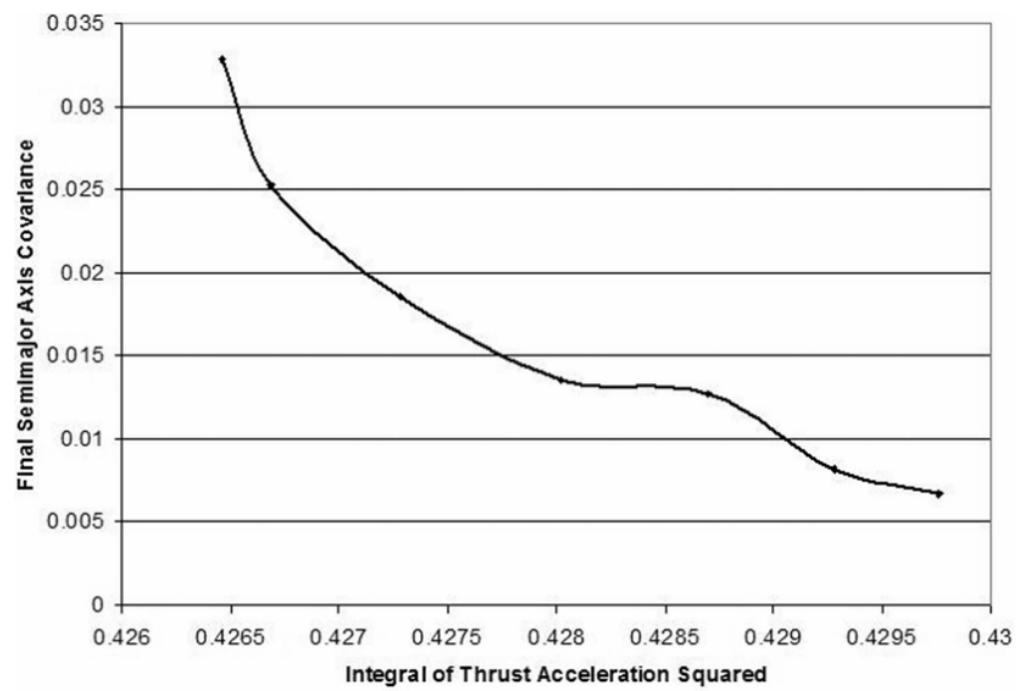

Fig. 13. Pareto optimal front.

Table VII Semimajor Axis Covariance and Thrust Acceleration for Near GEO to Lunar Orbit Transfers

\begin{tabular}{rcccc}
\hline \hline$\varphi * 10^{-4}$ & $\begin{array}{c}\text { Integral of Thrust Acc. } \\
\text { Squared } * 10^{-1}\end{array}$ & $\begin{array}{c}\text { Change in Integral of } \\
\text { Thrust Acc. Squared } * 10^{-5}\end{array}$ & $\begin{array}{c}\text { Semimajor Axis Cov. at } \\
\text { Final Time } * 10^{-3}\end{array}$ & $\begin{array}{c}\text { Change in Semimajor } \\
\text { Axis Cov. } * 10^{-3}\end{array}$ \\
\hline 0 & 4.2646 & N/A & 32.82 & N/A \\
3 & 4.2668 & 22 & 25.32 & -7.50 \\
6 & 4.2728 & 82 & 18.56 & -14.26 \\
9 & 4.2802 & 156 & 13.53 & -19.29 \\
12 & 4.2870 & 224 & 12.72 & -20.10 \\
15 & 4.2928 & 282 & 8.14 & -24.68 \\
18 & 4.2976 & 330 & 6.68 & -26.14 \\
\hline
\end{tabular}

The mass optimal transfer, with $\varphi$ equal to zero, and the transfer, with $\varphi$ equal to $1.8 * 10^{-4}$, that optimize for fuel consumption and observability are shown in Figs. 14 and 15. Despite the fact that the covariance associated with the semimajor axis is reduced significantly, the transfer trajectory is not altered significantly.

IEEE Transactions on Aerospace and Electronic Systems, Vol 46, No. 2 (April 2010): 771-791. DOI. This article is (C) Institute of Electrical and Electronics Engineers (IEEE) and permission has been granted for this version to appear in e-Publications@Marquette. Institute of Electrical and Electronics Engineers (IEEE) does not grant permission for this article to be further copied/distributed or hosted elsewhere without the express permission from Institute of Electrical and Electronics Engineers (IEEE). 


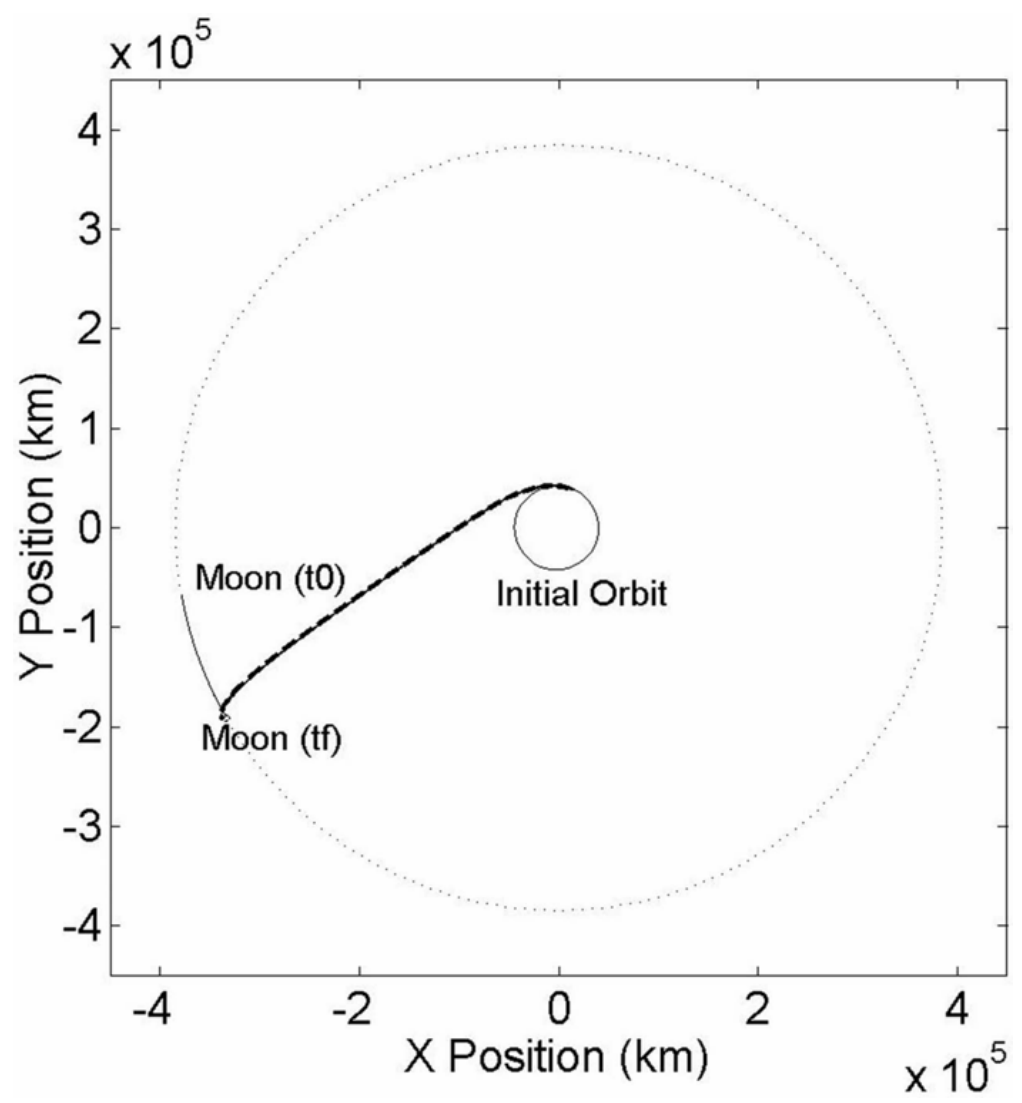

Fig. 14. Earth orbit to lunar orbit transfer.
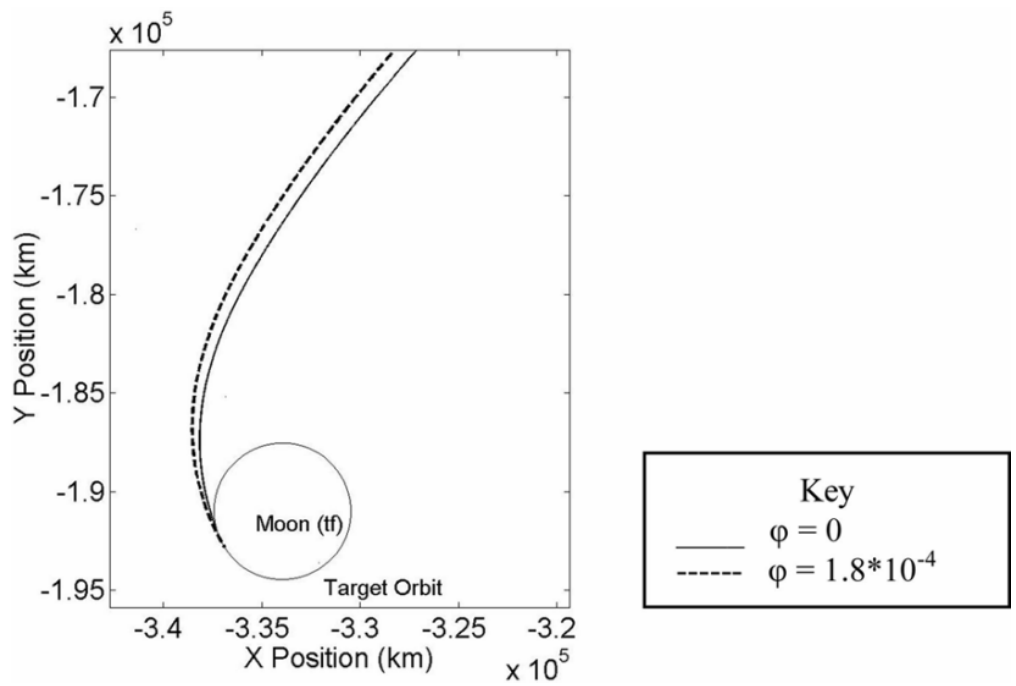

Fig. 15. Earth orbit to lunar orbit transfer (magnified).

Fig. 16 shows the magnitude of the thrust acceleration for the mass optimal transfer. Figs. 17 and 18 show the change in the thrust acceleration magnitude and the thrust

IEEE Transactions on Aerospace and Electronic Systems, Vol 46, No. 2 (April 2010): 771-791. DOI. This article is @ Institute of Electrical and Electronics Engineers (IEEE) and permission has been granted for this version to appear in e-Publications@Marquette. Institute of Electrical and Electronics Engineers (IEEE) does not grant permission for this article to be further copied/distributed or hosted elsewhere without the express permission from Institute of Electrical and Electronics Engineers (IEEE). 
direction when $\varphi$ changes from 0 to $1.8 * 10^{-4}$. Notice that the thrust acceleration magnitude is altered by between one-tenth of one percent and 10 percent during different periods of the transfer.

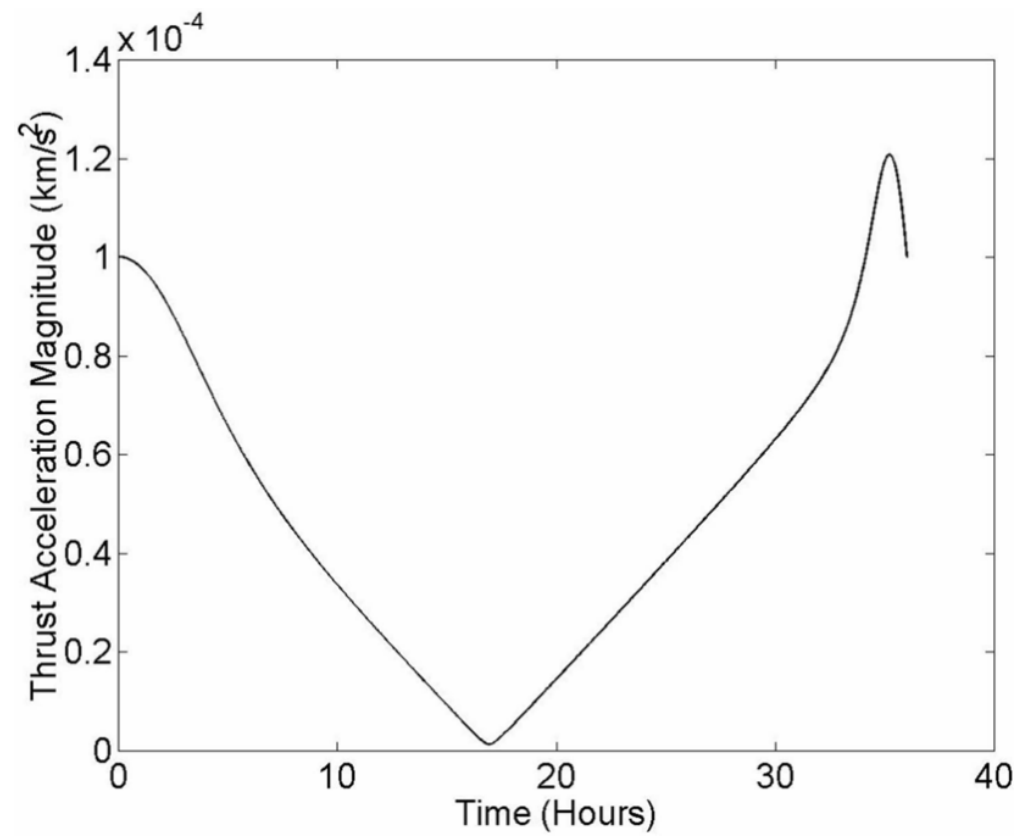

Fig. 16. Thrust acceleration magnitude on mass optimal transfer versus time.

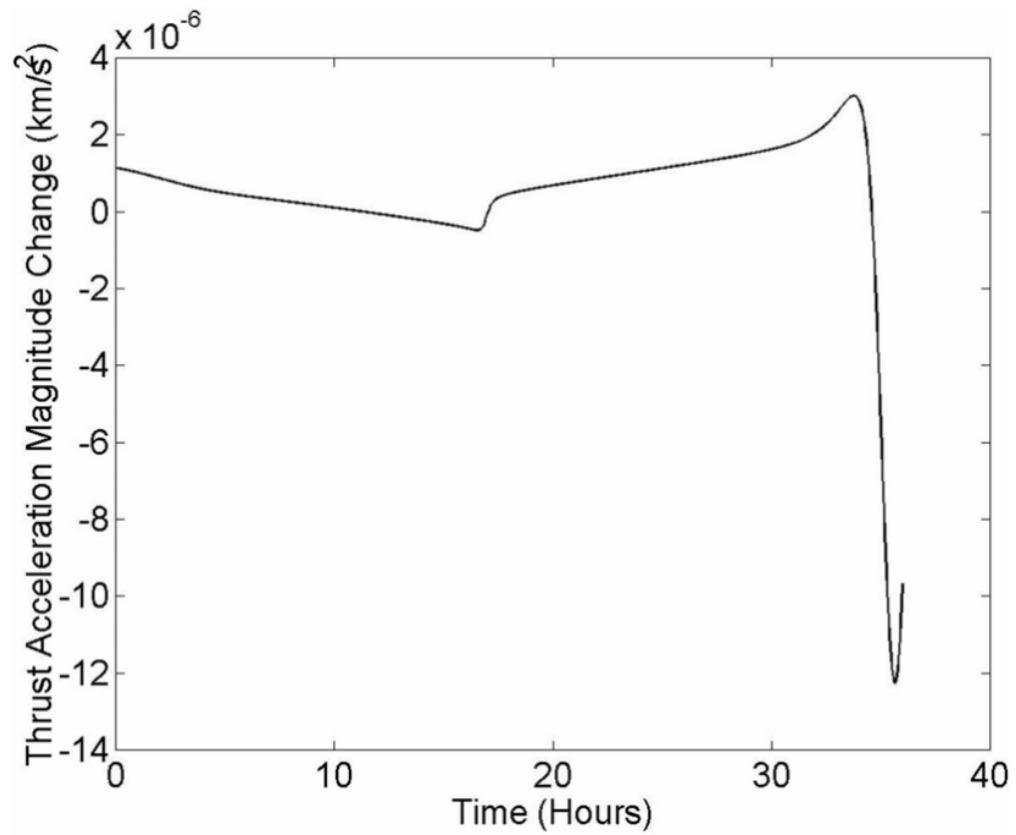

Fig. 17. Change in thrust acceleration magnitude between transfer with $\varphi=0.00018$ and mass optimal transfer versus time.

IEEE Transactions on Aerospace and Electronic Systems, Vol 46, No. 2 (April 2010): 771-791. DOI. This article is @ Institute of Electrical and Electronics Engineers (IEEE) and permission has been granted for this version to appear in e-Publications@Marquette. Institute of Electrical and Electronics Engineers (IEEE) does not grant permission for this article to be further copied/distributed or hosted elsewhere without the express permission from Institute of Electrical and Electronics Engineers (IEEE). 


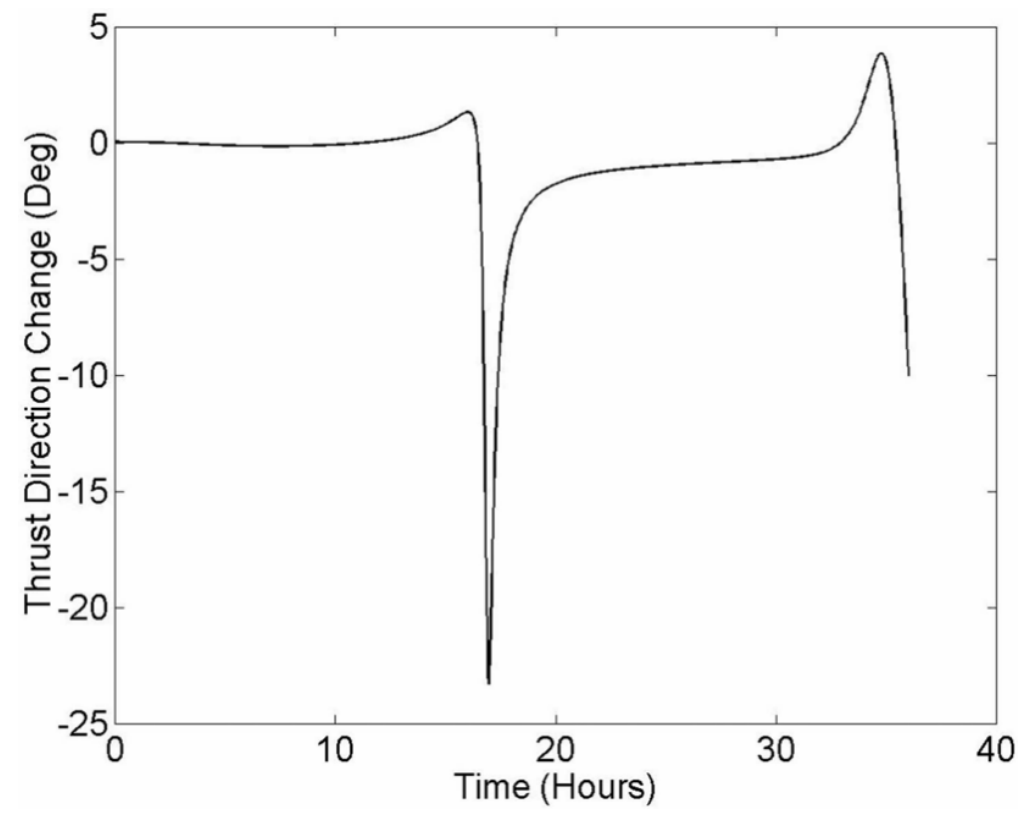

Fig. 18. Change in thrust direction between transfer with $\varphi=0.00018$ and mass optimal transfer versus time.

The covariance associated with the spacecraft semimajor axis, with respect to the Moon, along these two trajectories is shown in Fig. 19. This covariance is only plotted near the end of the trajectory because the spacecraft semimajor axis relative to the Moon does not have a significant meaning until the spacecraft approaches the Moon.

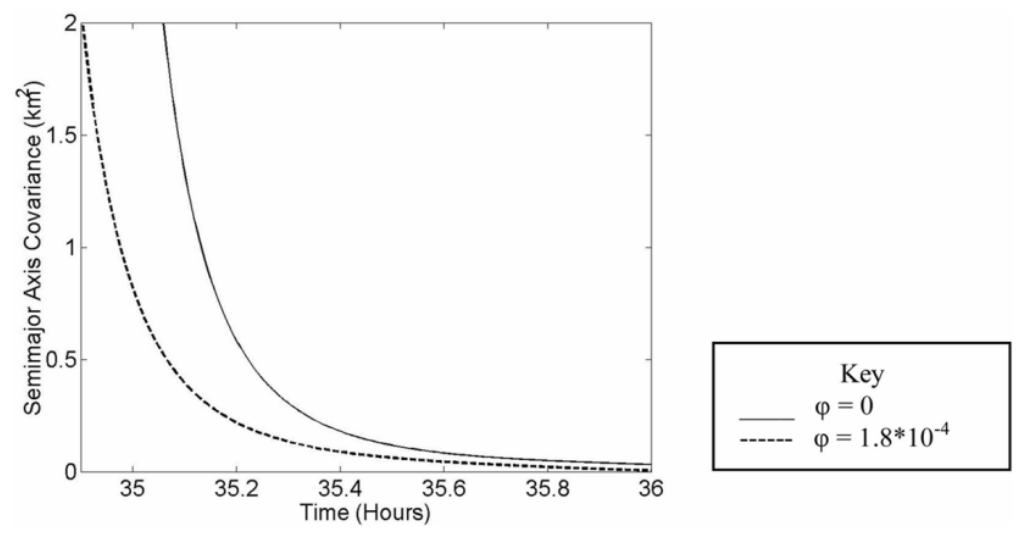

Fig. 19. Covariance associated with semimajor axis of the target lunar orbit versus time.

The costate associated with $P_{y y}$ is plotted in Fig. 20. Notice how this costate is largely unchanged during the early part of the trajectory and then changes a great deal as the spacecraft approaches the Moon. These large changes near the end of the trajectory

IEEE Transactions on Aerospace and Electronic Systems, Vol 46, No. 2 (April 2010): 771-791. DOI. This article is C Institute of Electrical and Electronics Engineers (IEEE) and permission has been granted for this version to appear in e-Publications@Marquette. Institute of Electrical and Electronics Engineers (IEEE) does not grant permission for this article to be further copied/distributed or hosted elsewhere without the express permission from Institute of Electrical and Electronics Engineers (IEEE). 
make it difficult to determine the initial value of these covariance costates because a very small change in the initial value of the costate or numerical integration error during the long portion of the trajectory where the costate remains nearly constant can cause a large violation of the constraint on these costates at the final time. These difficulties can be significantly reduced by including the value of the costate at an intermediate time in the unknown parameter list and by constraining the value of the costate before and after this intermediate time to be the same. Fig. 21 shows the costate associated with $P_{y y}$ near the end of the transfer for various values of $\varphi$. Notice that for this transfer from Earth orbit to lunar orbit, the covariance costates do not exhibit the same phase and amplitude behavior as they exhibit in previous transfers. The covariance costates do not simply increase in amplitude as the observability terms are weighted more heavily in the cost function.

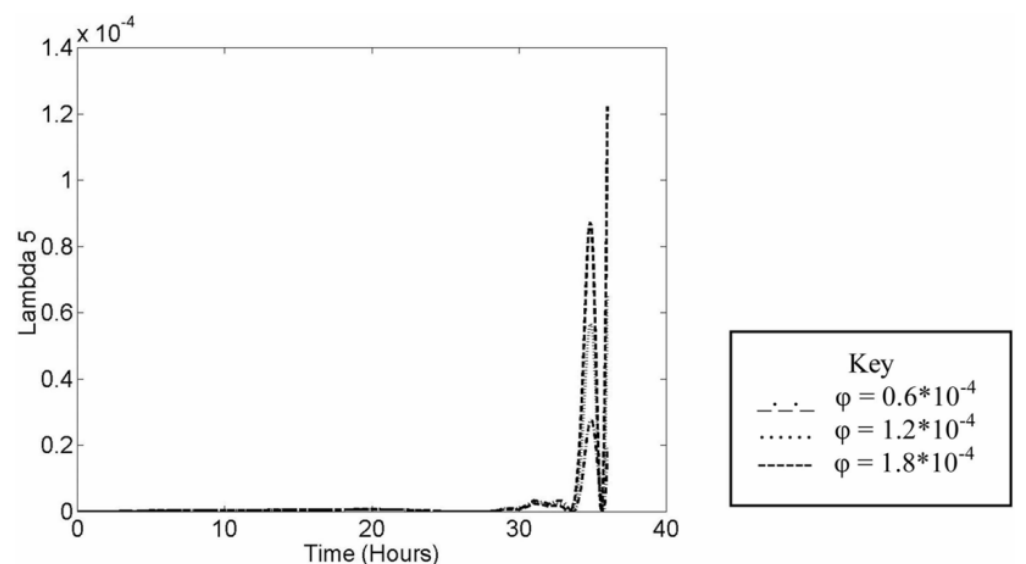

Fig. 20. Covariance costate associated with $P_{y y}$ versus time.

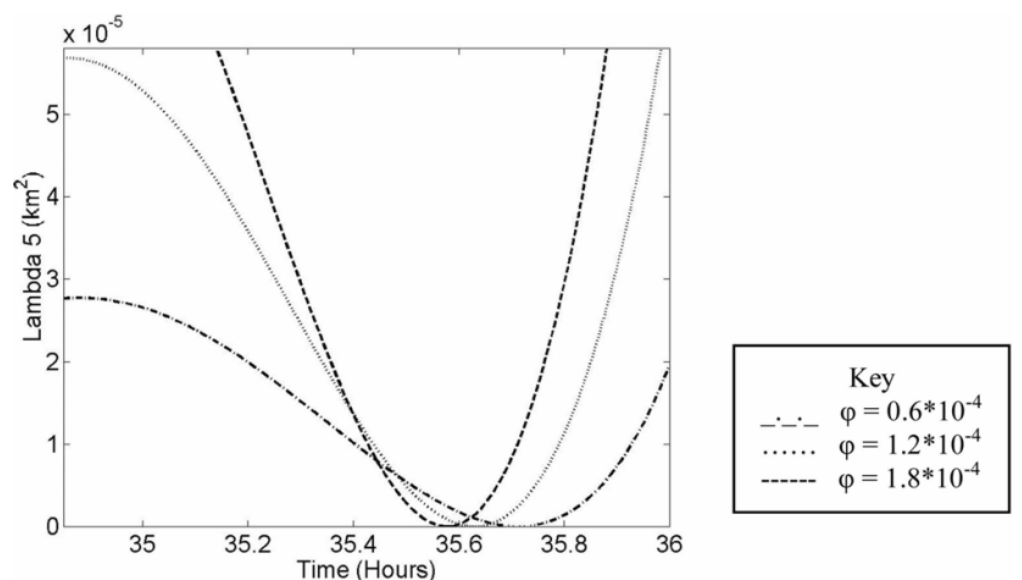

Fig. 21. Covariance costate associated with $P_{y y}$ versus time (magnified).

IEEE Transactions on Aerospace and Electronic Systems, Vol 46, No. 2 (April 2010): 771-791. DOI. This article is @ Institute of Electrical and Electronics Engineers (IEEE) and permission has been granted for this version to appear in e-Publications@Marquette. Institute of Electrical and Electronics Engineers (IEEE) does not grant permission for this article to be further copied/distributed or hosted elsewhere without the express permission from Institute of Electrical and Electronics Engineers (IEEE). 
NOT THE PUBLISHED VERSION; this is the author's final, peer-reviewed manuscript. The published version may be accessed by following the link in the citation at the bottom of the page.

\section{SECTION V.}

\section{Conclusion}

Adding either the trace of the covariance at discrete times or the integral of the trace of the covariance to the cost function allows one to determine trajectories with significantly improved observability with very little additional propellant required. These results indicate that the cost of further improving the observability increases as observability is increased.

The amount of fuel required to improve the observability along a trajectory by a given percentage depends on numerous factors, including the initial and final orbits as well as the transfer time. This research does not attempt to determine which trajectories require less propellant to improve observability. The models for the error in observations and errors in dynamic equations also affect the results. The effect of changing the ratio of terms in $\mathbf{R}, \mathbf{Q}$, and $\mathbf{P}\left(t_{0}\right)$ has not been investigated. Additionally these results assume scalar range measurements. Transitioning to different measurement types, multiple observers, or missions between different orbits may yield different results.

The differences between the optimal trajectories with, continuous measurements throughout the trajectory, alternating periods of continuous measurements and no measurements, and discrete measurements, are minimal for the example transfer from LEO to GEO. The optimal control histories and optimal costates are also similar for these three types of measurements. The results indicate that the exact times of the observations do not need to be known a priori in order to determine the fuel cost of decreasing the covariance by a given amount using this technique. The results do indicate that one must know the quality and quantity of the measurements that are taken. Additionally this study only examines trajectories in which the temporal spacing of the observations is equal. Further study is necessary to determine the effect of different measurement spacing.

The similarity between the results using discrete observations and the results using continuous observations indicates that the continuous model is sufficient to determine optimal trajectories, including observability. While one could theoretically use the method presented in this paper to solve an optimization problem with any number of discrete observations, one major difficulty limits the practical application of this method. In order to solve the TPBVP, one must compute the derivatives of the states and costates at the final time with respect to the costates at the initial time. As the number of observations increases, the number of jumps in the augmented state increases as well. These jumps in 
the state elements and the corresponding jumps in the costate elements make it difficult to compute the derivatives with enough accuracy to determine optimal trajectories with thousands of discrete measurements. Therefore the similar results between discrete and continuous observations indicate the continuous model should be used in order to avoid the numerical difficulties of the discrete model, unless an extremely high level of accuracy is necessary.

For the case of dynamic errors based on the thrust, the fact that it is more difficult to reduce the integral of the trace of the covariance by changing the thrust profile should be expected. When the engine thrusts more in an effort to place the spacecraft on a trajectory that is more observable, this process introduces more error into the equations of motion (because the model for dynamic error assumes that the error is proportional to thrust magnitude), thus causing the uncertainty to increase.

The example transfer from Earth orbit to lunar orbit illustrates one of the important conclusions about designing orbits to increase knowledge of the spacecraft state at the end of the transfer. The trajectory where spacecraft knowledge is improved spends a longer time near the Moon, where the gradient of the gravitational forces from the Moon can help distinguish the trajectory. It is easier to distinguish between two trajectories, when the gradient of the equations of motion with respect to the state is larger.

\section{References}

1J. T. Betts, "Survey of numerical methods for trajectory optimization", Journal of Guidance Control and Dynamics, vol. 21, pp. 193-207, 1998.

2D. E. Lawden, Optimal Trajectories for Space Navigation, London:Butterworth, 1963.

3D. J. Jezewski, Primer vector theory and applications, 1975.

${ }^{4}$ D. R. Vander Stoep, "Trajectory shaping for the minimization of state-variable estimation errors", IEEE Transactions on Automatic Control, vol. AC-13, pp. 284-286, 1968.

${ }^{5} \mathrm{G}$. T. Schmidt, A new technique for identification and control of systems with unknown parameters, 1970.

6J. L. Speyer, D. G. Hull, C. Y. Tseng, S. W. Larson, "Estimation enhancement by trajectory modulation for homing missiles", Journal of Guidance Control and Dynamics, vol. 4, pp. 167-174, 1984.

7D. G. Hull, J. L. Speyer, C. Y. Tseng, "Maximum-information guidance for homing missiles", Journal of Guidance Control and Dynamics, vol. 8, no. 4, pp. 494-497, 1985.

8D. G. Hull, J. L. Speyer, D. B. Burris, "Linear-quadratic guidance law for dual control of homing missiles", Journal of Guidance Control and Dynamics, vol. 13, no. 1, pp. 137-144, 1990.

9Y. Oshman, P. Davidson, "Optimization of observer trajectories for bearings-only target localization", IEEE Transactions on Aerospace and Electronic Systems, vol. 35, no. 3, pp. 892-902, 1999. 
${ }^{10} \mathrm{H}$. Seywald, R. Kumar, "Desensitized optimal trajectories", AIAA/AAS Spaceflight Mechanics Meeting, 1996.

${ }^{11} \mathrm{H}$. Seywald, "Desensitized optimal trajectories with control constraints", AIAA/AAS Spaceflight Mechanics Meeting, 2003.

12H. Seywald, "Desensitized optimal orbit insertion", AAS/AIAA SpaceFlight Mechanics Meeting, 2004.

13D. Mishne, "Guidance law for the reconfiguration of satellite formation in the presence of measurement errors", AIAA/AAS Astrodynamics Specialist Conference, 2005.

14]. R. Carpenter, E. R. Schiesser, "Importance of semi-major axis knowledge in the determination of near-circular orbits", Spaceflight Mechanics 1999, vol. 102, pp. 1297-1310, 1999.

15D. G. Hull, Optimal Control Theory for Applications, New York:Springer, 2003.

16R. H. Bishop, P. D. Burkhart, S. W. Thurman, "Observability during planetary approach navigation", Spaceflight Mechanics 1993, vol. 82, pp. 973-984, 1993.

17J. E. Prussing, "Equation for optimal power-limited spacecraft trajectories", Journal of Guidance Control and Dynamics, vol. 16, no. 2, pp. 391-393, 1993.

18A. Gelb, Applied Optimal Estimation, MA, Cambridge:Massachusetts Institute of Technology, 1989.

19S. Zimmer, C. Ocampo, R. Bishop, "Decreasing semimajor axis uncertainty through trajectory design", AIAA/AAS Astrodynamics Specialist Conference, 2005.

20S. Zimmer, C. Ocampo, "The use of analytical gradients to calculate optimal gravity assist trajectories", Journal of Guidance Control and Dynamics, vol. 28, no. 2, pp. 324-332, Mar.-Apr. 2005.

21S. Zimmer, C. Ocampo, "Analytical gradients for gravity assist trajectories using constant specific impulse engines", Journal of Guidance Control and Dynamics, vol. 28, no. 4, pp. 753-760, JulyAug. 2005.

${ }^{22}$ L. C. W. Dixon, M. C. Biggs, "The advantages of adjoint-control transformations when determining optimal trajectories by Pontryagin's maximum principle", Aeronautical Journal, pp. 169-174, Mar. 1972.

${ }^{23 K}$ K. P. Zondervan, L. J. Wood, T. K. Caughey, "Optimal low-thrust three-burn orbit transfers with large plane changes", The Journal of the Astronautical Sciences, vol. 32, no. 3, pp. 407-127, 1984. 